\title{
Distribution de l'avifaune des milieux forestiers de substitution (plantation et jachères) au Sud du Bénin en relation avec les facteurs de l'habitat
}

\author{
Toussaint O. LOUGBEGNON $^{{ }^{1 *}}$, Jean Claude T. CODJIA ${ }^{2}$ et Roland M. LIBOIS ${ }^{3}$ \\ 1, 2 Laboratoire d'Aménagement des Forêts et de Biogéographie (LAFBio/FSA/UAC); tlougbe@yahoo.fr ; \\ jtccodjia@yahoo.fr \\ ${ }^{3}$ Laboratoire de zoogéographie, Institut de Botanique (Université de Lièges, Belgique); \\ Roland.libois@ulg.ac.be \\ Auteur correspondant; E-mail : tlougbe@yahoo.fr
}

\section{RESUME}

Dans le Sud du Bénin, l'intensification de la récente déforestation par l'agriculture et l'urbanisation fait que les jachères et les plantations occupent plus de superficies que les forêts. Ainsi, ces agroécosystèmes sont à long terme susceptibles de remplacer les lambeaux forestiers. Pour tester la valeur conservatoire de ces milieux de substitution, les oiseaux ont été utilisés en tant qu'indicateur biologique en se basant sur une Analyse des Correspondances Multiples (ACM) et une Classification Hiérarchique Ascendante (CHA). 9 groupes écologiques d'oiseaux distribués dans les différents types de jachères et plantations ont été identifiés. Les jachères concentrent une forte proportion de l'avifaune de ces habitats de substitution forestiers. Ces résultats montrent que pour conserver la biodiversité des oiseaux dans ces habitats de substitution des forêts, les jachères sous plantations ou évoluées sont indispensables.

(C) 2010 International Formulae Group. All rights reserved.

Mots clés : Distribution, oiseaux, groupe écologique, plantation, jachère, Sud du Bénin.

\section{INTRODUCTION}

Les oiseaux sont très dépendants des facteurs de l'habitat et des variations de l'habitat. A ce titre, ils sont de bons indicateurs des changements écologiques (Martin et Thibault, 1983; Prodon, 1988; Bibby et al., 1992 ; Bersier et Meyer, 1995 ; Skowno et Bond, 2003; Gottschalk et al., 2007 ). C'est ainsi que les oiseaux sont souvent utilisés comme indicateurs écologiques soit pour caractériser les milieux, soit pour mesurer l'évolution des habitats (Anderson et Robbins, 1981; Bellamy et al., 2000). Compris comme tel, l'avifaune peut être valablement utilisée comme élément de typologie et de biomonitoring dans les milieux de substitution forestiers (jachère et plantation) au sud du Bénin qui, sont des parcelles de reconstitution forestière de différents âges issues de la déforestation. Ces deux types de milieux, constituent l'essentiel de la «couverture végétative » actuelle pour la faune en dehors des quelques lambeaux forestiers. Il est inévitable que les îlots forestiers existant ne peuvent plus à eux seuls contribuer à la conservation de l'avifaune de la région aujourd'hui. Des études écologiques sur l'avifaune pouvant aider à formuler des diagnostics en matière de conservation et d'aménagement font défaut surtout dans ces biotopes de substitution car, les quelques études ornithologiques des milieux terrestres existantes (Berlioz, 1956; Brunel, 1958 ; Coubéou, 1995 ; Anciaux, 1996, 2000 ; 
Walther, 1998; Van den Akker, 2003 ; Lougbégnon, 2002) n'en ont jamais spécifiquement fait cas de l'avifaune de ces milieux de substitution forestiers. Pour remédier à cette situation, cette étude sur l'avifaune, en relation avec les structures de l'habitat dans ces milieux, est menée dans l'optique de comprendre la répartition des communautés d'oiseaux des différents milieux de substitution, en réponse à la déforestation récente. Ainsi, les oiseaux de tous les types de milieux de substitution du sud du Bénin que sont jachères naturelles ou sous plantations et les plantations agroforestières de cocotier (Cocos nucifera), de teck (Tectona grandis) et de palmier à huile (Elaeis guineensis) ont été inventoriés afin de savoir (i)- Comment l'avifaune est distribuée dans ces plantations et les jachères?, (ii)- Quelles sont les variations de composition et de distribution des oiseaux entre ces deux types d'habitat? (iii)- Quels sont les groupes écologiques constitués à travers les différents milieux en présence ? et (iv)- S'il existe un classement des oiseaux en relation avec les facteurs de ces deux types de milieux?

Les réponses à ces interrogations sont nécessaires pour formuler les aménagements qui satisferont de façon durable la conservation de la nature.

\section{MATERIEL ET METHODES Milieu d'étude}

L'aire de l'étude se situe entre la côte atlantique et le septième parallèle de latitude Nord. Elle concerne la partie subéquatoriale du Bénin ou le domaine guinéen (Figure 1). Elle appartient au couloir de sécheresse appelé «Dahomey-Gap ». La température moyenne annuelle est de $27,5{ }^{\circ} \mathrm{C}$. Sur la base de la répartition des précipitations, on distingue deux saisons pluvieuses et deux saisons sèches intercalées. Les majeures parties des pluies sont concentrées entre les mois d'avril et de juin. Les précipitations moyennes annuelles varient entre 1000 à $1500 \mathrm{~mm}$. L'hygrométrie moyenne est toujours supérieure à 60\% (Akoegninou, 2004).
La couverture forestière de cette zone est constituée de forêts denses semi-décidues et savanes dérivées (Akoegninou, 2004). Mais aujourd'hui, cette zone est devenue la plus urbanisée du pays et, est caractérisée par une forte densité humaine estimée à plus de la moitié de la population béninoise (environ 4 millions) (500 habitants $/ \mathrm{km}^{2}$ ). Cette pression démographique explique la dégradation ou la disparition des formations forestières naturelles pour laisser place à diverses sortes de cultures et des plantations. Le paysage actuel offre donc de maigres bouquets de forêts denses humides semi-décidues dites forêts sacrées qui sont des reliques de l'ancienne formation forestière. A cela s'ajoutent, les galeries forestières des réseaux hydrographiques, les forêts marécageuses, les forêts reliées aux argiles noires de la dépression de la Lama.

\section{Matériel d'étude}

Le matériel de terrain est constitué d'une paire de jumelles, d'un télescope, le guide d'identification des oiseaux (Borrow et Demey, 2001), de la série des disques des chants des oiseaux africains (Chappuis, 2000), de guide d'identification de la flore analytique du Bénin (Akoegninou, 2006) et d'un GPS (Global Positioning System).

\section{Méthode de collecte des données \\ Choix des sites et stations d'étude}

L'étude s'est déroulée en divers siteséchantillons à la fois dans les jachères et les plantations dominantes du sud du Bénin. Au total 25 stations d'écoute fixes dont 13 dans les jachères et 12 dans les plantations sont régulièrement prospectées entre 2003 et 2008. Le Tableau 1 présente la description des stations échantillonnées.

Technique d'échantillonnage de l'avifaune et mode opératoire d'inventaire des oiseaux

La méthode de relevé de données d'avifaune appliquée est basée essentiellement sur des points d'écoute (Bibby et al., 1992). C'est l'échantillonnage de type ponctuel simple (EPS) précédée d'une mémorisation des chants des oiseaux. L'échantillonnage de type ponctuel simple est axé sur des points d'écoute 
de 20 minutes dans un rayon large (Cordonnier, 1976; Spitz, 1982; Prodon, 1988). Cette technique a l'avantage d'être à la confluence de deux autres méthodes classiques: celle des Indices Ponctuels d'Abondance (Blondel et Frochot, 1970) et celle des Echantillonnages Fréquentiels Progressifs (E.F.P) (Blondel, 1975). Cette méthode n'étant pas basée sur un prélèvement, mais sur la notation de contacts, donne d'excellents résultats dans l'étude de la structure du peuplement avien de différentes séries de végétation (Blondel et al., 1970).

A l'intérieur des 45 points d'écoute fixes (rayon de $150 \mathrm{~m}$ ), les oiseaux sont inventoriés pendant 20 minutes. L'observation ou la détection des oiseaux se fait par le chant, le cri ou à vue. Avant d'opérer, l'observateur reste immobile pendant 5 minutes au centre de ce cercle afin d'atténuer l'effet de sa présence sur les oiseaux. Tous les oiseaux vus posés (à terre ou dans les arbres) sont comptés, de même que les hirondelles, les martinets et les Accipitridae ou Falconidae (espèces qui chassent au vol) sont retenues. Les chants et les cris qui se retrouvent loin du cercle d'écoute ne sont pas pris en compte. Les relevés mentionnent les espèces contactées (inventaire systématique).

La fréquence de passage dans une même station est calquée aux rythmes saisonniers. L'essentiel des séances de prospection est effectuée pendant le jour. Les observations débutent à 6 heures et prennent fin le matin à 13 heures. Le soir, elles reprennent à 15 heures pour finir à 18 heures 30 ou 19 heures selon la saison.

Pour toute la période d'étude, 182 relevés ont été enregistrés au sein des stations de jachères et 650 relevés dans les stations de plantations.

\section{Technique d'échantillonnage des paramètres de l'habitat}

Les paramètres de l'habitat collectés sont le taux de recouvrement des strates de la végétation et la nature du sol. L'estimation du recouvrement des différentes strates est faite visuellement. Trois strates ont été distinguées : strate herbacée ou parfois buissonnante $(0 \mathrm{~m} \geq$ $\mathrm{Hh} \leq 3 \mathrm{~m})$, strate arbustive $(3 \mathrm{~m} \geq \mathrm{Ha} \leq 15 \mathrm{~m})$ et la strate ligneuse ou arborescente $(\mathrm{Hi} \geq 15 \mathrm{~m})$ (Sokpon, 1995 et Akoègninou, 2004). Quant à la nature du sol, quatre modalités sont associées à cette variable : sol sableux (SS), sol argilosableux ou ferralitique (SA), vertisol (SV) et sol hydromorphe $(\mathrm{SH})$. Ces variables sont identifiées à la même échelle que les peuplements d'oiseaux, sur la superficie d'un cercle de 150 mètres de rayon.

\section{Méthode d'analyse des données collectées}

L'analyse des correspondances multiples (ACM) a été utilisée pour l'ordination de l'avifaune à partir de leurs stations et réciproquement (Prodon 1988, Prodon et Lebreton 1994). Son utilisation permet de dégager des espèces indicatrices ou caractéristiques. Elle produit des supports d'expression des classifications (des cartes factorielles) et constitue une excellente méthode permettant de positionner les espèces et relevés sur des gradients écologiques (Chessel et Debouzie, 1983).

Les inventaires avifaunistiques étant faits dans différents milieux et que les relevés (avifaune-station d'écoute) sont de type taxonvariables du milieu, il est alors possible d'ordonner les stations d'après les espèces d'oiseaux qu'elles contiennent en note de présence-absence (Tableau 2) et de le soumettre en ACM. Toute espèce d'oiseau contactée est notée 1 dans les différentes stations et dans le cas contraire, elle est notée 0 .

Pour mieux objectiver la représentation des espèces et des stations tel que cela apparaît dans le plan euclidien, les 6 premières coordonnées des axes des espèces et des stations dans le plan euclidien issues de l'ACM sont retenues pour une Classification Ascendante Hiérarchique de Ward. En effet, cette classification permet de regrouper les espèces et les facteurs de l'habitat qui tendent à se retrouver ensemble dans les mêmes relevés, donc ayant des traits écologiques voisins. En clair, l'objectif d'une telle analyse est de dégager les « groupes écologiques » d'oiseaux de telle ou telle station.

Les différents relevés sont encodés à l'aide du tableur Excel, 2000 pour être 
facilement exportés vers le logiciel XLSTATPro 7.5 pour les différentes analyses.

\section{Analyse effectuée sur les résultats}

Pour une meilleure lisibilité des graphiques d'ACM, les variables à très faibles ou à très fortes modalités sont éliminées de l'opération (ou sont regroupées avec d'autres modalités) grâce à un tri à plat des occurrences des oiseaux dans les stations. Ceci ne modifie pas sensiblement les résultats de l'analyse du fait de leurs pondérations. Ainsi, on a trié et éliminé certaines espèces de la matrice 118 espèces x 25 stations pour restreindre et alléger la matrice d'analyse. Ce sont les espèces d'oiseaux qui apparaissent dans 1, 2, 24 et 25 stations. L'intérêt de cette opération est de rendre fluide la lecture des cartes factorielles. Ce sont :

$>$ Espèces apparaissant dans une seule station: Polyboroides typus (Pot), Poicephalus senegalus (Pos), Clamator levaillantii (Cll), Coracias cyanogaster (Coc), Smithomis capensis (Smc), Lamprotornis splendidus (Las),

$>$ Espèces apparaissant dans 02 stations : Butorides striatus (Bus), Gypohierax angolensis (Gya), Glaucidium perlatum (Glp), Andropadus virens (Anv), Chlorocichla flavicollis (Chf), Nicator chloris (Nic) ;

$>$ Espèces apparaissant dans 24 stations : Apus affinis (Apf) ;

$>$ Espèces apparaissant dans 25 stations : Bulbucus ibis (Bui), Milvus migrans (Mim), Centropus senegalensis (Ces), Dendropicos goertae (Deg), Hirundo semirufa (Hie), Hirundo senegalensis (Hin), Pycnonotus barbatus (Pyb), Turdus pelios (Tup), Turdoides plebejus (Tul), Corvinella corvina (Cor), Tchagra senegala (Tcs), Ploceus nigerrimus (Pli), Ploceus cucullatus (Plu).

De même, les données relatives aux facteurs de l'habitat (paramètre du milieu) sont ensuite codées suivant un mode binaire $(0,1)$ pour être facilement exploitables. Ainsi, pour la couverture de la végétation, ont reçu le code du chiffre 1 les variables : couverture en herbacée ( $\mathbf{C H H})$, couverture en arbuste (CHA), couverture des arbres (CHI) $>10 \%$. Toutes les autres variables stationnelles ayant des valeurs inférieures sont affectées du chiffre 0 comme code. De même, les différentes modalités de la variable «sol» sont codées en présence-absence.

Pour vérifier l'existence d'un certain degré de liaison entre les espèces et les données décrivant les stations, nous avons réalisé une analyse des correspondances multiples (ACM) séparément sur chacun des relevés ornithologiques et variables de milieu, puis nous avons calculé le coefficient de corrélation linéaire entre les coordonnées des stations sur les premiers axes factoriels issus de ces analyses. L'équation du modèle de la distribution s'écrit: $\mathrm{F} 1=0,957 * \mathrm{~F} 1$. Le coefficient obtenu pour $\mathrm{F} 1$ oiseaux/F1 milieu ( $\mathrm{r}=0,76$ et $p<0,0001$ ) est statistiquement significatif comme l'indiquent la Figure 2.

On peut donc affirmer qu'il existe un degré de liaison certain entre la composition de l'avifaune des stations étudiées et les variables de milieu que nous avons prises en considération. De ce fait, une nouvelle ACM reprenant à la fois les données ornithologiques et les données relatives à l'habitat a été réalisée.

\section{RESULTATS}

Composition et richesse spécifique des espèces d'oiseaux des milieux prospectés

$\mathrm{Au}$ total, 118 espèces sont observées dans l'ensemble des deux types de milieux (Tableau 2).

Analyse de la distribution des groupes écologiques des stations et des oiseaux dans le plan euclidien

Carte factorielle et valeur propre des axes

Les Figures 3 et 4 présentent la carte factorielle obtenue. Les valeurs propres extraites par les trois premiers axes factoriels sont respectivement de $23 \%, 17 \%$ et $10,60 \%$ correspondant ainsi à une inertie totale de $50,98 \%$. Ce qui est suffisant pour tirer des conclusions majeures. Dans la suite, l'interprétation des données va se limiter à ces trois axes. 


\section{Caractérisation écologique des stations dans le plan euclidien}

5 groupes de stations apparaissent dans le plan F1 x F2 (Figure 3). On a respectivement :

- le groupe 1 des plantations de Cocos nucifera ( $\mathrm{C} 1, \mathrm{C} 2, \mathrm{O} 1$ et $\mathrm{O} 2)$ situé à l'extrémité positive de F1-F2,

- le groupe 2 des plantations de Tectona grandis (T1, T2, T3 et T4) situé à l'extrémité positive de $\mathrm{F} 1$ et à l'extrémité négative de F2. La station $\mathrm{T} 1$ est toutefois un peu désolidarisée des autres,

- le groupe 3 formé par les plantations d'Elaeis guinneensis (E1, E2, P1, P2) (extrémités négative $\mathrm{F} 1$ et positive $\mathrm{F} 2$ ),

- le groupe 4 des stations de jachères sousplantation évoluée et jachères anciennes naturelles (jachères arbustives) (D1, D2, A2, 11 et 12), localisé de part et d'autre de l'extrémité négative de F2. Les positions très décalées des stations $\mathrm{A} 2$ et 12 suggèrent des différences évidentes ;

- le groupe 5 des jachères buissonnantes ou herbacées (D3, D4, A1, A3, A4, A5, O3 et O4) situé aux extrémités négatives des deux axes. La position proche axiale des groupes 3 et 5 traduit des stations des milieux à faciès intermédiaire entre les jachères et les plantations.

Enfin, F1 oppose les plantations de Cocos nucifera et de Tectona grandis aux jachères herbacées ou buissonnantes avec un important tassement des stations de jachères à l'extrémité négative de cet axe. F2 par contre, est un axe de séparation des plantations de Tectona grandis des autres types de plantations (cocoteraie et palmeraie).

Dans le plan de F1xF3, (Figure 4) on remarque que la structure étoilée de la projection des stations obtenue dans le plan F1-F2 prend une disposition en arche du nuage des points de présence (parabole convexe). Ceci résulte de la nette prépondérance de l'axe F1. En effet, l'inertie de F1 est de $22,71 \%$ contre $10,33 \%$ pour F3. La contribution de F3 est alors faible pour influencer la partition de la carte. Néanmoins, F3 oppose nettement les plantations d'Elaeis guineensis et jachères arbustives aux plantations de Cocos nucifera et de Tectona grandis. F1-F3 met en évidence le sixième groupe de station de jachère herbacée (O3, $\mathrm{O} 4, \mathrm{~A} 3$ ) issu de la dislocation des jachères buissonnantes et herbacées.

Interprétation des groupes écologiques des stations issus de la $\mathrm{CAH}$

En considérant le dendrogramme des stations (Figure 5) à hauteur de $\mathrm{D}=16$, on obtient 6 groupes de stations bien hiérarchisés. Ainsi, les stations de plantation de Cocos nucifera $(\mathrm{C} 1, \mathrm{C} 2, \mathrm{O} 1, \mathrm{O} 2)$ forment le premier groupe qui se démarque des autres types de milieux. Le deuxième groupe est formé par les plantations d'Elaeis guinneensis (P1, P2, E1, $\mathrm{E} 2)$. Le troisième groupe est celui des jachères ouvertes (herbacée) (A1, A2, D3, D4, A3). Le quatrième groupe est constitué par les stations de Tectona grandis (T1, T2, T3, T4).

Le cinquième groupe est constitué par les jachères arbustives $(11,12, \mathrm{D} 1, \mathrm{D} 2)$. Le sixième groupe est une association des jachères de grandes couvertures herbacée et arbustive (A4, A5, O3, O4).

En conclusion, 6 groupes de stations apparaissent dans les 2 plans. Il existe une nette individualisation au sein des stations de cocoteraie et de teckeraie.

\section{Caractérisation écologique des oiseaux dans le plan euclidien}

F1 oppose les oiseaux de milieux boisés et très couverts $(\mathrm{CHl})$, sur vertisol $(\mathrm{SV})$ situés à droite à l'extrémité positive de cet axe à ceux des milieux buissonnants, herbacés (CHH) sur sols ferralitique ou hydromorphe ( $\mathrm{SA}$ et $\mathrm{SH}$ ) situé à l'extrémité négative de cet axe (Figure 6). Cet axe traduit à la fois un gradient de la structure de la végétation et de pédologie. De nombreuses espèces sont inféodées à ces caractéristiques écologiques issues de l'axe F1. Il s'agit de :

- Halcyon malimbica (Ham), Lybius bidentatus (Lyb), Cisticola anonymus (Cin), Falco curvieri (Fac), Chrysococcyx cupreus (Chc), Pogoniulus bilineatus (Pob), Eurystomus glaucurus (Eug), Sylvietta virens (Syv), Muscicapa striata (Mus), Anthus leucophrys (Anl), Cinnyris chloropygius (Cic), Phylloscopus trochillus (Pht), Phoeniculus purpureus (Php), Cisticola 
brachypterus (Cib), Cinnyris cupreus (Ciu), Passer griseus (Pag), Cinnyris coccinigaster (Cio), Corvus albus (Coa), Uraeginthus bengalus (Urb), Cinnyris venustus (Civ), Hirundo rustica (Hir), Chalcomitra senegalensis (Che), Lagonosticta senegala (Lae). Ils forment le groupe des oiseaux des milieux boisés et couverts car la position correspondant à leur point de présence est en effet rejetée à droite de l'axe. Ce sont là surtout des oiseaux des plantations de Tectona grandis et accessoirement des autres stations boisées.

- Un deuxième groupe montrant également la même tendance avec F1 mais un peu moins accusée est constitué par le tassement important proche de l'origine par les espèces comme: Saxicola rubetra (Sar), Chrysococcyx klass (Chk), Prinia subflava (Prs), Falco biarmicus (Fab), Vidua chalybeata (Vic), Kaupifalco monogramicus (Kam), Ploceus superciliosus (Plp), Streptopelia vinacea (Stv), Prionops plumatus (Prp), Lonchura bicolor (Lob), Sylvia Borin (Syb), Euplectes hordeaceus (Euh), Streptopelia senegalensis (Ste), Macrodipterix longipennis (Mal) Hirundo abyssinica (Hia), Apus apus (Apa), Euplectes macrourus (Eum), Cypsiurus parvus (Cyp) Plocepasser superciliosus (Pls), Camaroptera brachyura (Cab). Ce sont des espèces de jachères arbustives et parfois ubiquistes entre les milieux boisés en générale (plantations et jachères boisées).

En ce qui concerne F2, on remarque que cet axe oppose les oiseaux de Cocos nucifera et de d'Elaeis guinneensis à ceux de Tectona grandis. La position très marquée des points de présence sur cet axe des espèces aviennes comme Alcedo leucogaster (All), Anthreptes gabonicus (Anb), Lamprotornis purpureus (Lap), Ploceus aurantius (Pla), confirme que c'est un axe de présence de l'avifaune surtout rencontrée dans les plantations de Cocos nucifera. La variable sol sableux (SS) est à cet effet la seule qui ait contribué à l'édification de F2.

Certains oiseaux comme Macronyx croceus (Mac), Ptilostomus afer (Pta), Tockus nasitus (Ton), Dendropicos pyrrhogaster
(Dep), Hirundo smithii (His), Thescelocichla leucopleura (Thl), Turtur tympanistria (Tut), Passer domesticus (Pad), Psittacula krameri (Psk), Anthreptes collaris (Anc), Nigrita canicapilla (Nia), Ceuthmochares aereus (Cea), Cyanomitra obscura (Cyo), Accipiter badius (Acb), Dicrurus adsimilis (Dia) et de Petronia dentata (Ped) représentés sur F2 mais moins marqués que les précédents (proches de l'origine des axes) constituent le groupe des espèces de plantations d'Elaeis guinneensis.

A ces 4 groupes, s'ajoute un cinquième groupe formé par l'association de Caprimulgus inormatus (Cai), Quelea erythrops (Que), Falco ardosiaceus (Faa), Turtur abyssinicus (Tub), Ploceus melanocephalus (Plm), d'oiseaux savanicoles rares, contactées surtout dans les jachères herbacées ou buissonnantes et sous-bois herbacé de plantations de palmier à huile sur sols ferralitique ou hydromorphe (SA et $\mathrm{SH}$ ) et qui, sont représentées à l'extrémité négative de F1 x F2. F3 est un axe de grande couverture en herbacée ou buissonnante (CHH) (Figure 7). Cette situation présente clairement à l'extrémité positive de F3 le groupe des espèces d'oiseaux de jachère herbeuse et de sous-bois herbeux de palmier comme Caprimulgus inormatus (Cai), Euplectes franciscanus (Euf), Falco ardosiaceus (Faa), Hippolais pallida (Hip), qui ont le plus contribué à l'édification de F3.

La disposition en parabole des points de présence des oiseaux dans F1-F3 imposée par F1 ne permet pas à F3 de marquer une nette opposition entre les oiseaux des différents habitats, mais on note tout de même une opposition entre les oiseaux des jachères herbacées à ceux des habitats boisés (plantations et jachères arbustives) que sont : Turtur tympanistria (Tut), Campethera caillautii (Cam), Macronyx croceus (Mac).

En outre, on retrouve aussi à l'extrémité positive de F1-F3 une bonne individualisation ( tassement important proche de l'origine) des oiseaux inféodés aux habitats de grandes couvertures arbustives que sont: Estrilda melpoda (Esm), Malimbus scutatus (Mas), Elanus caeruleus (Elc), Chalcomitra 
senegalensis (Che), Macrodipterix longipennis (Mal), Francolinus bicalcaratus (Frb), Euplectes macrourus (Eum), Crinifer piscator (Crp), Cypsiurus parvus (Сур), Plocepasser superciliosus (Pls), Lonchura cucullata (Loc).

Interprétation des groupes écologiques des oiseaux issus de la $\mathrm{CAH}$

Le dendrogramme des espèces d'oiseaux (Figure 8) à hauteur de $\mathrm{D}=2$, montre également 6 groupes d'avifaunes bien hiérarchisés (G1, G2, G3, G4, G5 et G6). Ainsi :

- groupe 1 est formé par 7 oiseaux caractéristiques des plantations de Tectona grandis : Tyto alba (Tya), Chrysococcyx klass (Chk), Falco curvieri (Fac), Chrysococcyx cupreus (Chc), Pogoniulus bilineatus (Pob), Eurystomus glaucurus (Eug) et Sylvietta virens (Syv) ;

- groupe 2 est celui des oiseaux ubiquistes entre plantations et jachères. Il est formé par 9 espèces : Myioparus plumbeus (Myp), Otus leucotis (Otl), Falco biarmicus (Fab), Saxicola rubetra (Sar), Muscicapa striata (Mus), Anthus leucophrys (Anl), Cinnyris chloropygius (Cic), Prinia subflava (Prs), Merops malimbicus (Mem) ;

- groupe 3 est une association de 12 oiseaux des plantations de Cocos nucifera et d'Elaeis guineensis, Ploceus aurantius (Pla), Alcedo leucogaster (All), Anthreptes gabonicus (Anb), Lamprotornis purpureus (Lap), Ptilostomus afer (Pta), Passer domesticus (Pad), Thescelocichla leucopleura (Thl), Macronyx croceus (Mac), Halcyon malimbica (Ham), Lybius bidentatus (Lyb), Cisticola anonymus (Cin), Phylloscopus trochillus (Pht); Chlorocichla simplex (Chs), Turtur tympanistria (Tut), Ispidina picta (Isp), Psittacula krameri (Psk) et Chrysococcyx caprius (Cha) ;

- groupe 4 est composé de 2 espèces d'oiseaux typiques des jachères arbustives: Turtur abyssinicus (Tub), Ploceus melanocephalus (Plm),

- groupe 5 est une association de 6 oiseaux des jachères buissonnantes ou de sous-bois buissonnants d'Elaeis guinnensis: Falco ardosiaceus (Faa), Vidua chalybeata (Vic),
Caprimulgus climacurus (Cal), Ploceus superciliosus (Plp), Cisticola brachypterus (Cib), Motacilla flava (Mof);

- groupe 6 est une association complexe de 51 espèces d'oiseaux :

ubiquistes entre jachères et plantations boisées : Campethera caillautii (Cam), Quelea erythrops (Que), Dendropicos pyrrhogaster (Dep), Hirundo smithii (His), Turtur afer (Tua), Malimbus scutatus (Mas), Streptopelia semitorquata (Sts), Elanus caeruleus (Elc), Prionops plumatus (Prp), Nigrita canicapilla (Nia), Anthreptes collaris (Anc), Accipiter badius (Acb), Ceuthmochares aereus (Cea), Cyanomitra obscura (Cyo), Petronia dentata (Ped), typiques de jachères herbacées: Vidua macroura (Vim), Passer griseus (Pag), Uraeginthus bengalus (Urb), Euplectes hordeaceus (Euh), Hirundo rustica (Hir), Lagonosticta senegala (Lae), Caprimulgus inormatus (Cai), Estrilda melpoda (Esm), Euplectes franciscanus (Euf), Francolin bicalcaratus (Frb), Centropus grillii (Ceg), Corvus albus (Coa), Streptopelia vinacea (Stv), Halcyon senegalensis (Has), Streptopelia senegalensis (Ste), Camaroptera brachyura $\mathrm{Cab}$ ), nettement ubiquistes entre tous les habitats et, dont la seule exigence est l'existence de couvert végétal disposant de quelques ligneux: Tockus nasitus (Ton), Phoeniculus purpureus (Php), Cinnyris cupreus (Ciu), Lonchura bicolor (Lob), Apus apus (Apa), Hippolais pallida (Hip), Macrodipterix longipennis (Mal), Cypsiurus parvus (Cyp), Cinnyris coccinigaster (Cio), Cinnyris venustus (Civ), Chalcomitra.

Enfin, à ces groupes s'ajoutent les espèces qui ont été supprimées lors de l'analyse du fait qu'elles n'apportent pas trop d'informations :

- les espèces apparaissant dans une seule station sont celles inféodées surtout aux plantations. On retrouve des espèces comme: Polyboroides typus (Pot), Poicephalus senegalus (Pos), Clamator levaillantii (Cll), Coracias cyanogaster (Coc), Smithomis capensis (Smc), Lamprotornis splendidus 


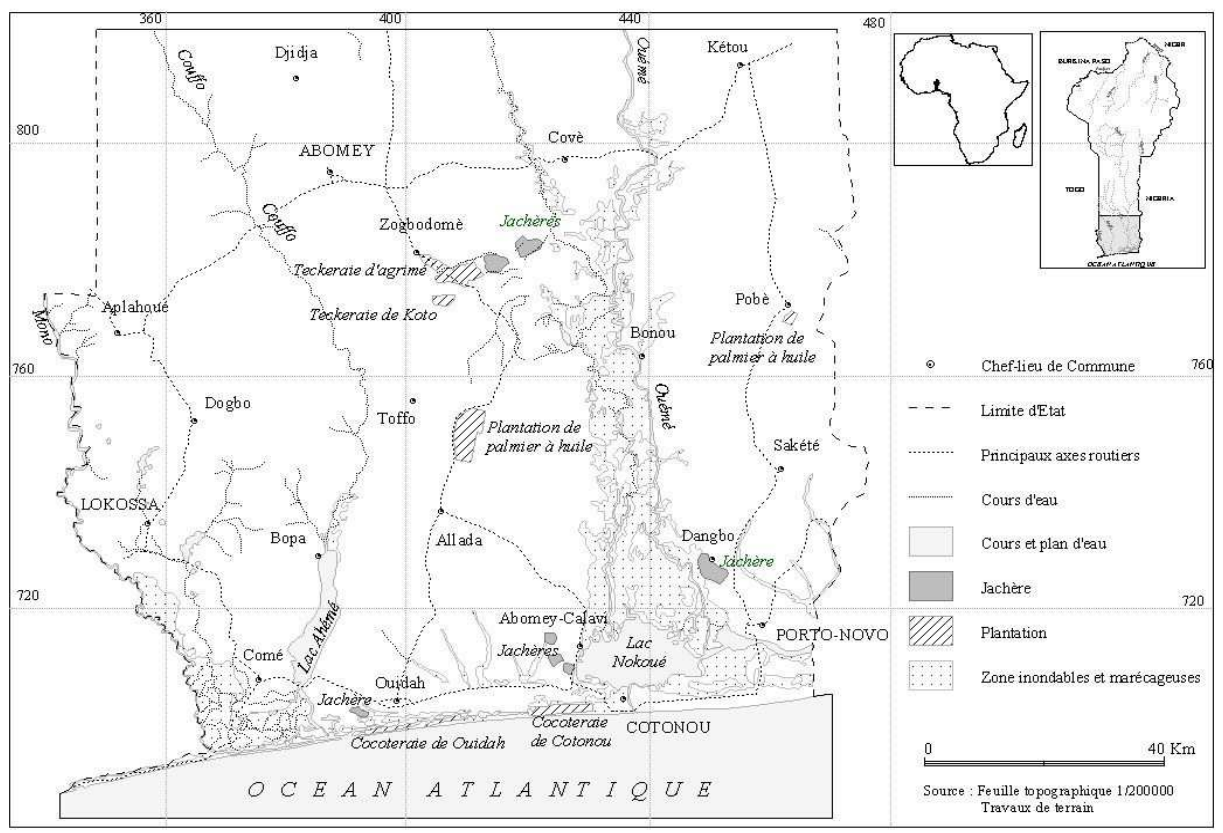

Figure 1: Cartes de localisation de la zone et des sites d'étude.

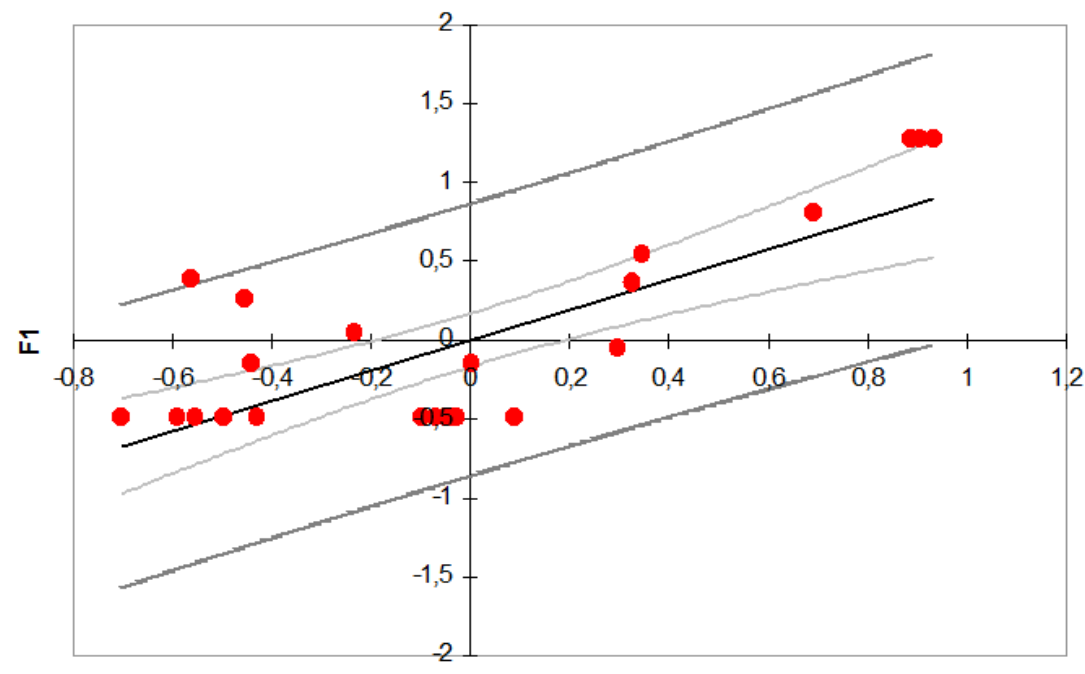

F1

\begin{tabular}{|cl|}
\hline - Individus & - Prédictions \\
\hline
\end{tabular}

Figure 2 : Droites F1/F1 de corrélation entre la position des stations sur les axes de l'ACM "oiseau" et de l'ACM "variables de l'habitat". 


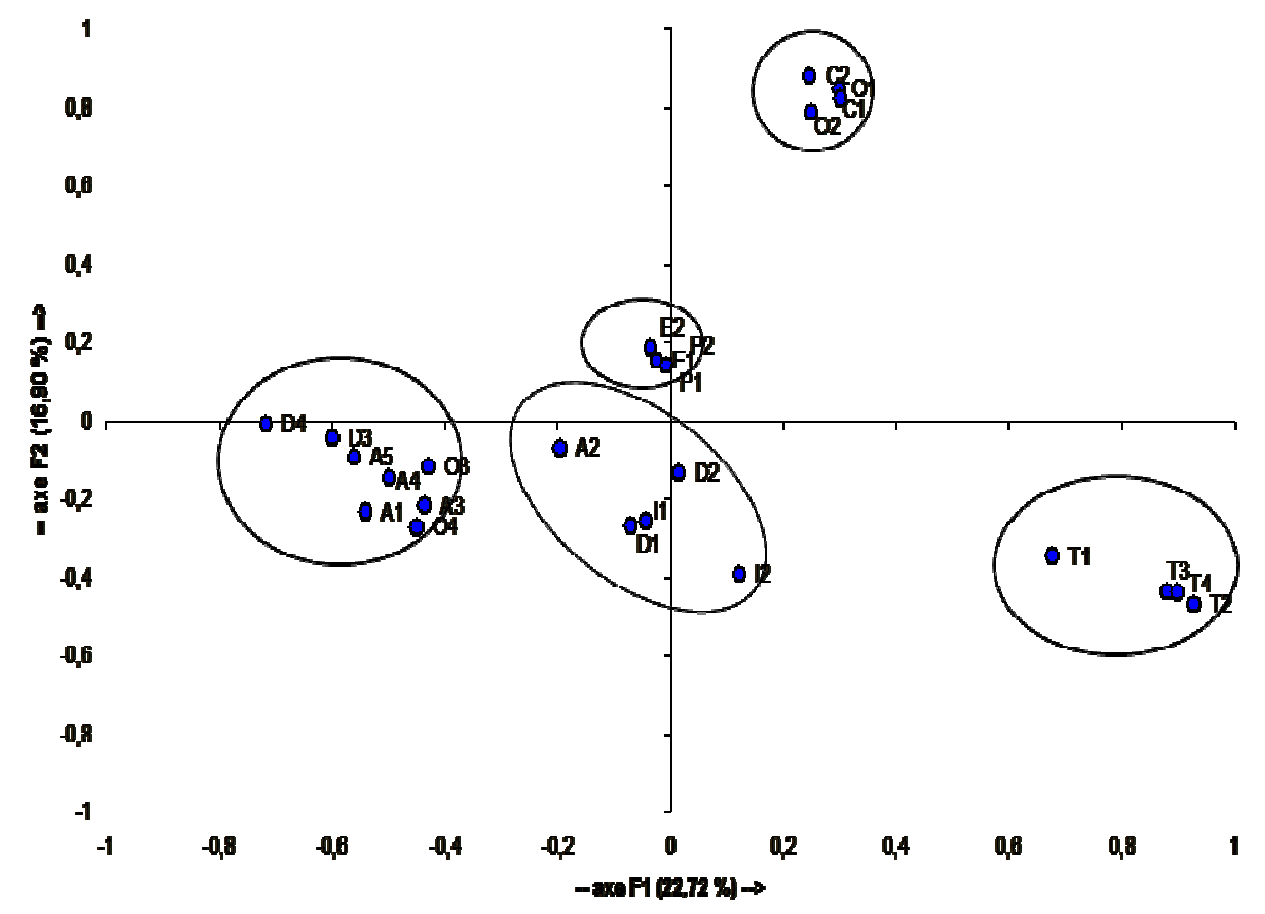

Figure 3 : Distribution des stations dans le plan factoriel (axes F1 et F2 : 39,62\%).

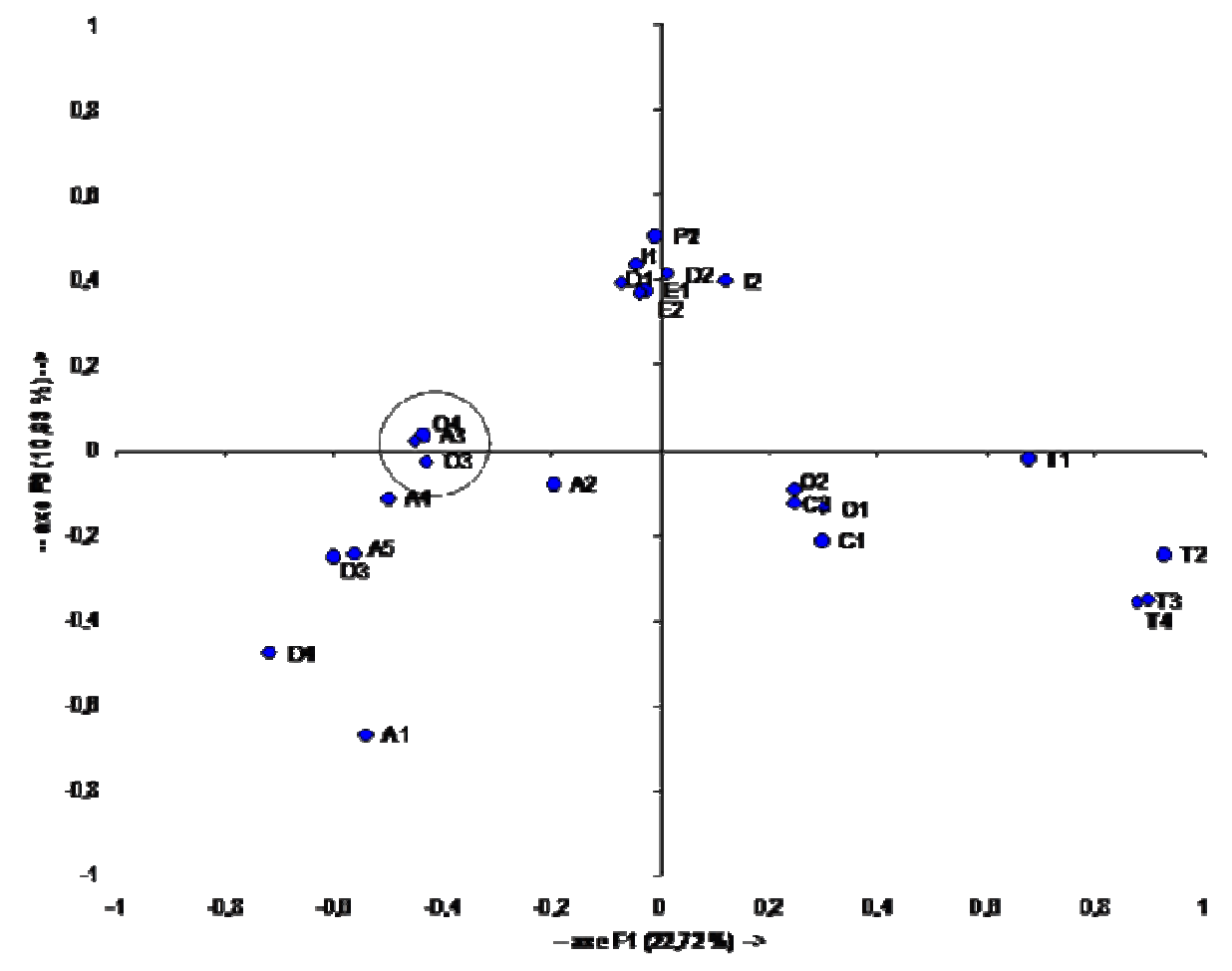

Figure 4 : Distribution des oiseaux dans le plan factoriel (axes F1 et F3 : 33,65\%). 


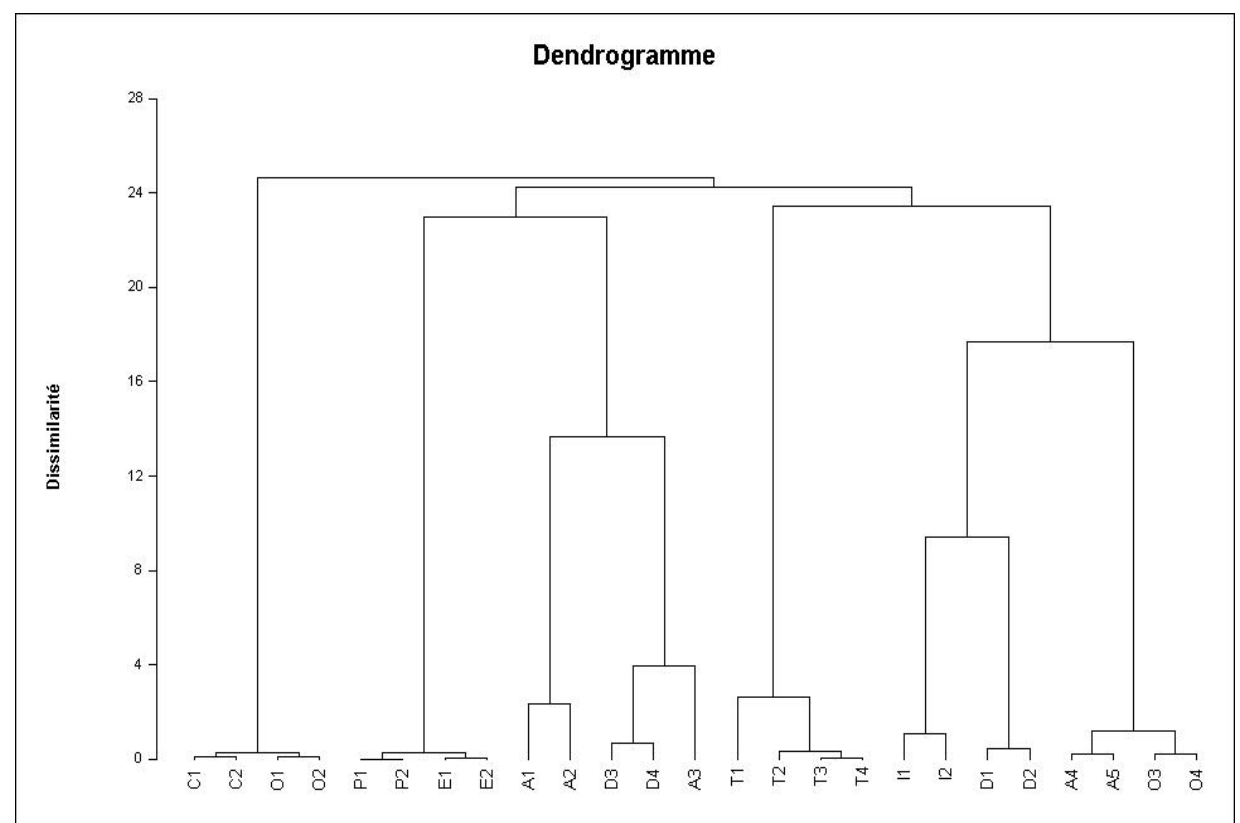

Figure 5 : Dendrogramme de dissimilarité de Ward des stations.

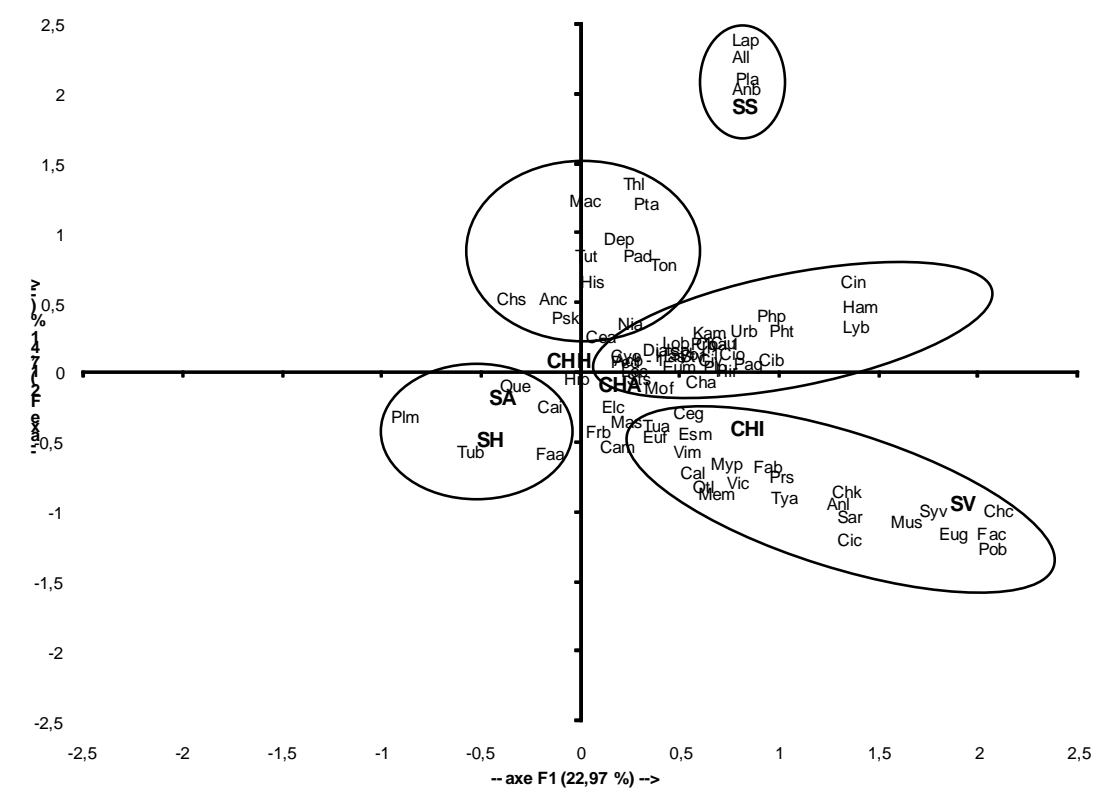

Figure 6 : Distribution des oiseaux dans le plan factoriel (axes F1 et F2 : 40,38\%). 


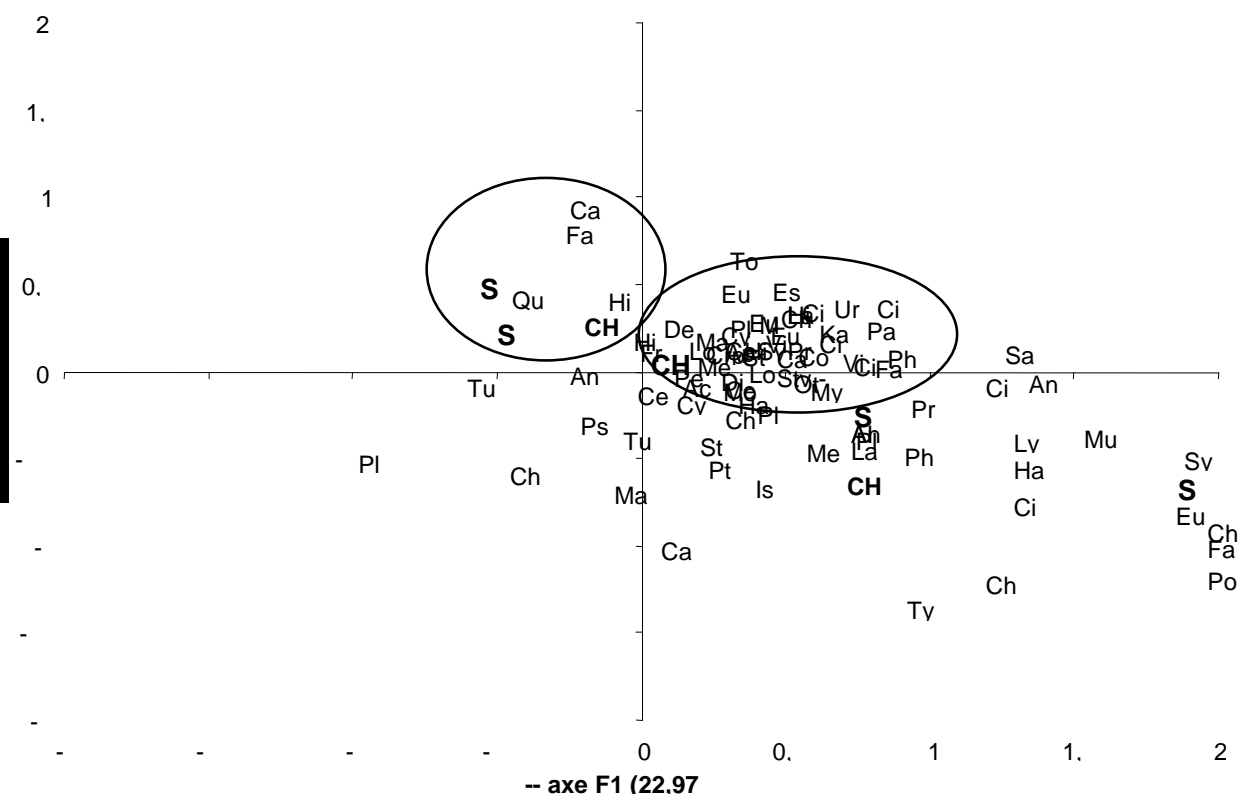

Figure 7 : Distribution des oiseaux dans le plan euclidien (axes F1 et F3 : 33,57\%).

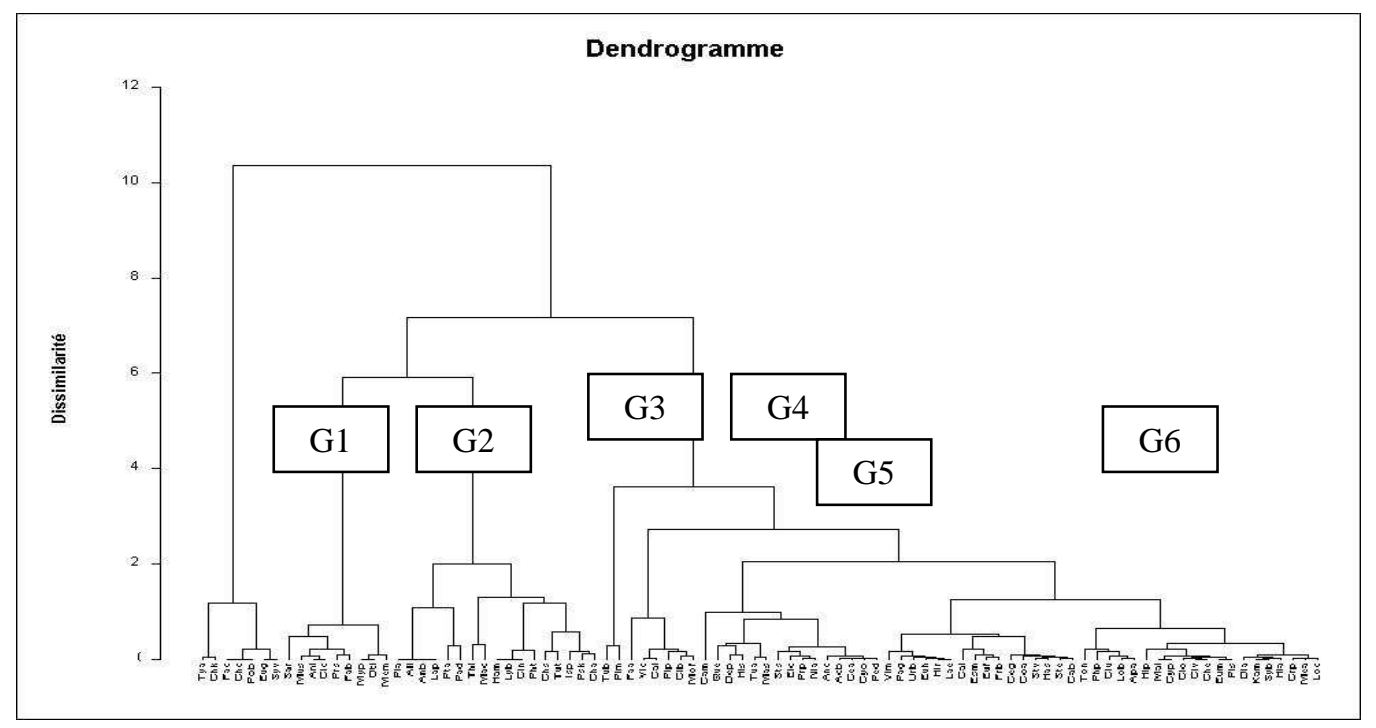

Figure 8 : Dendrogramme de dissimilarité des espèces d'oiseaux selon la méthode de Ward. 
Tableau 1 : Liste des stations et description de leurs paramètres.

\begin{tabular}{|c|c|c|c|c|}
\hline & & & Paramètres du milieu & \\
\hline Stations & Types d'écosystème & Strate arborescente & Strate arbustive & Strate herbacée ou sous arbustive\$ \\
\hline Station de Togbin $1(\mathrm{C} 1)$ & $\begin{array}{l}\text { vieille plantation de } 15 \text { ans } \\
\text { de Cocos nucifera }\end{array}$ & $\begin{array}{l}\text {-Hauteur des cocotiers } 16-18 \\
\text { m } \\
\text {-Recouvrement } 20 \%\end{array}$ & $\begin{array}{l}\text {-Cyperus maritimus, Remirea } \\
\text { maritima et de fourrés littoraux de } \\
\text { Chrysobalanus sp. de } 4 \mathrm{~m} \text { hauteur } \\
\text {-taux de recouvrement de } 50 \%\end{array}$ & $\begin{array}{l}\text {-quelques plages de strates sous-arbustives de } 30 \mathrm{~cm} \mathrm{de} \\
\text { haut faites de Tephrosia purpurea } \\
\text {-taux de recouvrement } 50 \%\end{array}$ \\
\hline Station de Togbin $2(\mathrm{C} 2)$ & $\begin{array}{l}\text { jeune plantation de } 6 \text { ans de } \\
\text { Cocos nucifera }\end{array}$ & $\begin{array}{l}\text {-des pieds de cocotier de } \\
\text { hauteur entre } 1,5 \mathrm{~m} \text { et } 3 \mathrm{~m} \\
\text {-recouvrement } 30 \%\end{array}$ & inexistante & $\begin{array}{l}\text {-sous-bois presque inexistant en saison sèche, et } \\
\text { constitués de champ de tomate (Lycopersicum } \\
\text { esculentum) de hauteur } 50 \mathrm{~cm} \\
\text {-recouvrement de } 60 \% \text { pendant la saison pluvieuse }\end{array}$ \\
\hline Station de Ouidah $1(\mathrm{O} 1)$ & $\begin{array}{l}\text { jeune plantation de } 6 \text { ans de } \\
\text { Cocos nucifera }\end{array}$ & $\begin{array}{l}\text { - } 6 \text { m environs de haut, } \\
\text { - taux de recouvrement } 10 \%\end{array}$ & inexistante & $\begin{array}{c}\text { - quelques plages de Tephrosia purpurea de } 30 \mathrm{~cm} \\
\text {-recouvrement n'excédant pas } 5 \%\end{array}$ \\
\hline Station de Ouidah $2(\mathrm{O} 2)$ & $\begin{array}{l}\text { vieille plantation de plus } 15 \\
\text { ans de Cocos nucifera }\end{array}$ & $\begin{array}{l}\text {-Cocos nucifera de hauteur } \\
15 \text { m environs } \\
\text {-taux de recouvrement } 15 \%\end{array}$ & $\begin{array}{l}\text {-sous-bois de Paspalum vaginatum } \\
\text { et Typha australis de hauteur } 1 \text { à } 2 \\
\mathrm{~m} \\
\text {-taux de recouvrement estimé à } \\
50 \%\end{array}$ & inexistante \\
\hline Station de Ouègbo 1 (E1) & $\begin{array}{l}\text { plantation d'Elaeis } \\
\text { guineensis }\end{array}$ & $\begin{array}{l}\text {-Elaeis guineensis de hauteur } \\
\text { variant entre } 10 \text { et } 13 \mathrm{~m} \\
\text {-recouvrement de } 30 \%\end{array}$ & inexistante & $\begin{array}{c}\text {-sous-bois de } 1,5 \mathrm{~m} \text { de Chromolaena odorata, } \\
\text {-taux de recouvrement de } 50 \%\end{array}$ \\
\hline Station de Ouègbo 2 (E2) & $\begin{array}{l}\text { plantation d'Elaeis } \\
\text { guineensis }\end{array}$ & $\begin{array}{l}\text {-Elaeis guineensis de hauteur } \\
\text { comprise entre } 10 \text { et } 13 \mathrm{~m} \\
\text {-taux de recouvrement } 30 \%\end{array}$ & inexistante & $\begin{array}{l}\text {-sous-bois de Chromolaena odorata de hauteur } 1,5 \text { et } 2 \\
\text { m } \\
\text {-recouvrement } 90 \% \text {. }\end{array}$ \\
\hline Station de Pobè $1(\mathrm{P} 1)$ & $\begin{array}{l}\text { vieille plantation de plus de } \\
20 \text { ans d'Elaeis guineensis }\end{array}$ & $\begin{array}{l}\text {-Elaeis guineensis de hauteur } \\
\text { avoisinant } 15 \mathrm{~m} \\
\quad \text {-recouvrement de } 20 \%\end{array}$ & inexistante & $\begin{array}{l}\text {-sous-bois de } 1,5 \mathrm{~m} \text { de haut dominé par Chromolaena } \\
\text { odorata, } \\
\text {-taux de recouvrement de } 50 \%\end{array}$ \\
\hline Station de Pobè 2 (P2) & $\begin{array}{l}\text { Plantation d'Elaeis } \\
\text { guineensis }\end{array}$ & $\begin{array}{l}\text { Elaeis guineensis de hauteur } \\
15 \mathrm{~m} \text { avec un taux de } \\
\text { recouvrement } 30 \%\end{array}$ & inexistante & $\begin{array}{c}\text {-sous-bois de Chromolaena odorata de hauteur } 1,5 \mathrm{~m} \\
\text {-recouvrement } 90 \% .\end{array}$ \\
\hline
\end{tabular}


T. O. LOUGBÉGNON et al. / Int. J. Biol. Chem. Sci. 4(4): 1191-1216, 2010

\begin{tabular}{|c|c|c|c|c|}
\hline Station d'Agrimey 1 (T1) & $\begin{array}{c}\text { jeune plantation de } 6 \text { ans de } \\
\text { Tectona grandis }\end{array}$ & $\begin{array}{l}\text { - Tectona grandis de plus de } \\
20 \text { m de hauteur } \\
\text { - recouvrement de } 50 \%\end{array}$ & $\begin{array}{l}\text { - les rejets de Tectona grandis de } \\
\text { hauteur } 2 \text { à } 3 \mathrm{~m} \\
\text {-recouvrement } 80 \%\end{array}$ & $\begin{array}{l}\text {-sous-bois de Chromolaena odorata et de Andropogon } \\
\text { sp. de } 1 \mathrm{~m} \text { de hauteur } \\
\text {-recouvrement } 30 \%\end{array}$ \\
\hline Station d'Agrimey 2 (T2) & $\begin{array}{l}\text { vieille plantation de Tectona } \\
\text { grandis datant de } 40 \text { ans }\end{array}$ & $\begin{array}{l}\text {-Tectona grandis de plus de } \\
20 \text { m de hauteur } \\
\text {-recouvrement variable entre } \\
5 \text { et } 20 \% \text { selon des saisons }\end{array}$ & $\begin{array}{l}\text { - les rejets de Tectona grandis ou } \\
\text { régénération naturelle de Khaya } \\
\text { senegalensis et Diospyros } \\
\text { mespilformis de hauteur } 2 \text { à } 3 \mathrm{~m} \\
\quad \text { - recouvrement } 80 \%\end{array}$ & $\begin{array}{l}\text {-sous-bois de } 1 \mathrm{~m} \text { de hauteur, composé par chromolaena } \\
\text { odorata et Anchomanes difformis } \\
\text {-recouvrement } 10 \%\end{array}$ \\
\hline Station de Koto 1 (T3) & $\begin{array}{l}\text { plantation jeune (10 ans) de } \\
\text { Tectona grandis, bordée de } \\
\text { rideau de Gmelina arborea }\end{array}$ & $\begin{array}{l}\text { - Gmelina arborea et de } \\
\text { Tectona grandis de } 20 \mathrm{~m} \\
\text { - recouvrement } 30 \%\end{array}$ & $\begin{array}{l}\text {-les rejets de Tectona grandis de } \\
\text { hauteur } 2 \text { à } 3 \mathrm{~m} \\
\quad \text { - recouvrement } 20 \% \\
\end{array}$ & $\begin{array}{l}\text { - Chromolaena odorata et Andropogon sp. de } 1 \mathrm{~m} \mathrm{de} \\
\text { hauteur } \\
\text { - recouvrement } 10 \%\end{array}$ \\
\hline Station de Koto 1 (T3) & $\begin{array}{l}\text { plantation jeune }(10 \text { ans }) \text { de } \\
\text { Tectona grandis, bordée de } \\
\text { rideau de Gmelina arborea }\end{array}$ & $\begin{array}{l}\text { - Gmelina arborea et de } \\
\text { Tectona grandis de } 20 \mathrm{~m} \\
\text { - recouvrement } 30 \%\end{array}$ & $\begin{array}{l}\text {-les rejets de Tectona grandis de } \\
\text { hauteur } 2 \text { à } 3 \mathrm{~m} \\
\quad \text { - recouvrement } 20 \%\end{array}$ & $\begin{array}{l}\text { - Chromolaena odorata et Andropogon sp. de } 1 \mathrm{~m} \text { de } \\
\text { hauteur } \\
\text { - recouvrement } 10 \%\end{array}$ \\
\hline Station de Koto 2 (T4) & $\begin{array}{l}\text { plantation jeune (10 ans) } \\
\text { Tectona grandis }\end{array}$ & $\begin{array}{l}\text { - Tectona grandis } 20 \mathrm{~m} \\
\text { - recouvrement } 50 \% \text {; }\end{array}$ & $\begin{array}{l}\text {-les rejets Tectona grandis de } \\
\text { hauteur } 2 \text { à 3m -recouvrement } 20 \%\end{array}$ & $\begin{array}{l}\text {-Chromolaena odorata et Andropogon } s p \text { de } 1 \mathrm{~m} \text { de } \\
\text { hauteur } \\
\text { - recouvrement } 10 \%\end{array}$ \\
\hline Station de Egnonhlê (11) & $\begin{array}{l}\text { vieille jachère naturelle qui } \\
\text { subit tous les ans l'effet des } \\
\text { feux de végétation }\end{array}$ & inexistante & $\begin{array}{l}\text {-Lonchocarpus sericeus, Anogeissus } \\
\text { leiocarpa Ceiba pentandra et } \\
\text { Manguifera indica } 5 \text { à } 6 \mathrm{~m} \\
\text { - taux de recouvrement de } 30 \%\end{array}$ & $\begin{array}{l}\text { - sous-bois de } 1,5 \mathrm{~m} \text { de Chromolaena odorata } \\
\text { - recouvrement } 70 \%\end{array}$ \\
\hline Station de lokoli (12) & $\begin{array}{l}\text { vieille jachère naturelle } \\
\text { contiguë à la forêt } \\
\text { marécageuse de Lokoli }\end{array}$ & $\begin{array}{l}\text {-Anogeissus leiocarpa, } \\
\text { Adansonia digitata et Elaeis } \\
\text { guineensis } 5 \text { à } 15 \mathrm{~m} \\
\text { - taux de couverture de } 10 \% \\
\end{array}$ & 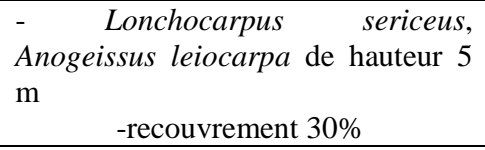 & $\begin{array}{c}\text { - strate sous-arbustive de } 1 \mathrm{~m} \text { de hauteur faite de } \\
\text { Chromolaena odorata et Imperata cylindrica } \\
\text { - recouvrement à 50\% }\end{array}$ \\
\hline Station de Dangbo 1 (D1) & plantation de Citrus sinensis & inexistante & $\begin{array}{l}\text { - Citrus sinensis de } 2,5 \mathrm{~m} \text { à } 3 \mathrm{~m} \text { de } \\
\text { hauteur } \\
\quad \text { - recouvrement par à } 20 \%\end{array}$ & $\begin{array}{l}\text { - strate herbacée de } 1 \mathrm{~m} \text { dominée par Andropogon } \\
\text { gayanus et Panicum sp. } \\
\text {-recouvrement de } 70 \% \text { à } 80 \% \text {. }\end{array}$ \\
\hline Station de Dangbo 1 (D1) & plantation de Citrus sinensis & inexistante & $\begin{array}{l}\text { - Citrus sinensis de } 2,5 \mathrm{~m} \text { à } 3 \mathrm{~m} \text { de } \\
\text { hauteur } \\
\quad \text { - recouvrement par à } 20 \%\end{array}$ & $\begin{array}{l}\text { - strate herbacée de } 1 \mathrm{~m} \text { dominée par Andropogon } \\
\text { gayanus et Panicum sp. } \\
\text {-recouvrement de } 70 \% \text { à } 80 \% \text {. }\end{array}$ \\
\hline Station de Dangbo 2 (D2) & jeune jachère de 4 ans sous & - Elaeis guineensis de $10 \mathrm{~m}$ & - Musa sp. 2 à $3 \mathrm{~m}$ & - Mucuna sp. de $1 \mathrm{~m}$ de hauteur \\
\hline
\end{tabular}


T. O. LOUGBÉGNON et al. / Int. J. Biol. Chem. Sci. 4(4): 1191-1216, 2010

\begin{tabular}{|c|c|c|c|c|}
\hline & $\begin{array}{c}\text { plantation d'Elaeis } \\
\text { guineensis contiguë au lit } \\
\text { d'inondation du fleuve } \\
\text { Ouémé } \\
\end{array}$ & $\begin{array}{l}\text { de hauteur } \\
\quad \text { - recouvrement de } 60 \%\end{array}$ & - $40 \%$ de couverture & - $100 \%$ de recouvrement \\
\hline Station de Dangbo 3 (D3) & $\begin{array}{l}\text { jeune jachère de } 4 \text { ans sous } \\
\text { plantation d'Elaeis } \\
\text { guineensis }\end{array}$ & $\begin{array}{l}\text { Elaeis guineensis de } 6 \mathrm{~m} \text { de } \\
\text { hauteur } \\
\quad \text { - recouvrement de } 50 \% \text {; }\end{array}$ & $\begin{array}{l}\text {-jeunes pousses de Eucalyptus } \\
\text { camaldulensis de } 3 \mathrm{~m} \text { de haut } \\
\text {-recouvrement de } 5 \%\end{array}$ & $\begin{array}{l}\text { strate herbacée de moins de } 1 \mathrm{~m} \text { dominée par Imperata } \\
\text { cylindrica } \\
\quad-70 \% \text { de recouvrement }\end{array}$ \\
\hline Station de Dangbo 4 (D4) & $\begin{array}{c}\text { une vieille jachère de } 10 \text { ans } \\
\text { environs }\end{array}$ & $\begin{array}{l}\text { - Eucalyptus camaldulensis } \\
\text { et Elaeis guineensis de } 5 \text { à } 6 \\
\text { m de haut } \\
\text { - recouvrement de } 90 \%\end{array}$ & Inexistante & $\begin{array}{c}\text { - sous-bois de hauteur } 1 \mathrm{~m} \text {, dominée par Chromolaena } \\
\text { odorata, Andropogon sp. et Tephrosia bracteolata } \\
\text { - taux de recouvrement de } 60 \%\end{array}$ \\
\hline Station de Yovocodji 1 (O3) & $\begin{array}{l}\text { jeune jachère de } 4 \text { ans sous } \\
\text { plantation d'Elaeis } \\
\text { guineensis }\end{array}$ & Inexistante & $\begin{array}{l}\text {-Elaeis guineensis de } 3 \mathrm{~m} \mathrm{de} \\
\text { hauteur } \\
\text { - recouvrement de } 50 \%\end{array}$ & $\begin{array}{c}\text {-strate herbacée de Tridax procumbens } \\
\text { d'Andropogon gayanus de } 30 \mathrm{~cm} \text { à } 1 \mathrm{~m} \text { de hauteur } \\
\text { - recouvrement } 90 \%\end{array}$ \\
\hline Station de Yovocodji 2 (O4) & $\begin{array}{c}\text { ancienne jachère de } 8 \text { ans } \\
\text { localisée dans une prairie } \\
\text { marécageuse de contact avec } \\
\text { la mangrove }\end{array}$ & Inexistante & $\begin{array}{l}\text {-Mitragyna inermis et Zanthoxyllun } \\
\text { zanthoxyloides hauteur } 5 \text { à } 6 \mathrm{~m} \\
\text { - recouvrement de } 50 \%\end{array}$ & $\begin{array}{l}\text { - strate herbacée de hauteur } 1 \mathrm{~m} \text { faite de Cyperus } \\
\text { articulatus et Andropogon gayanus } \\
\text {-recouvrement de } 80 \% \text {. }\end{array}$ \\
\hline $\begin{array}{c}\text { Station du Campus UAC } 1 \\
\text { (A1) }\end{array}$ & $\begin{array}{c}\text { ancienne jachère de } 7 \text { à } 8 \text { ans } \\
\text { de la portion arborée du } \\
\text { Nord du campus } \\
\text { universitaire d'Abomey- } \\
\text { Calavi }\end{array}$ & \begin{tabular}{l}
-Terminalia catapa \\
Terminalia superba \\
hauteur 12 à $16 \mathrm{~m}$ \\
\multicolumn{2}{c}{ de } \\
-recouvrement de $20 \%$
\end{tabular} & $\begin{array}{l}\text { - Delonix regia, Cocos nucifera et } \\
\text { Azadirachta indica de } 5 \text { à } 6 \mathrm{~m} \text { de } \\
\text { haut } \\
\text { - recouvrement de } 30 \%\end{array}$ & $\begin{array}{l}\text {-strate herbacée de Imperata cylindrica de } 0,5 \mathrm{~m} \mathrm{de} \\
\text { haut et très parcellaire } \\
\text {-recouvrement de } 10 \% \text {. }\end{array}$ \\
\hline Station de Zogbadjè (A2) & $\begin{array}{l}\text { jeune jachère sous plantation } \\
\text { d'Eucalyptus camaldulensis } \\
\text { et de Citrus sp }\end{array}$ & $\begin{array}{l}\text { Eucalyptus camaldulensis de } \\
\text { hauteur } 20 \mathrm{~m} \\
\quad \text {-recouvrement de } 30 \%\end{array}$ & $\begin{array}{l}\text {-quelques pieds d'Elaeis guineensis } \\
\text { et de Manguifera indica de hauteur } \\
6 \text { à } 10 \mathrm{~m} \\
\quad \text {-recouvrement de } 5 \% \text {; }\end{array}$ & $\begin{array}{c}\text {-sous-bois buissonnant de } 1 \mathrm{~m} \text { à } 2 \mathrm{~m} \text { de haut dominée } \\
\text { par Andropogon sp. et Chromolaena odorata } \\
\text {-recouvrement de } 100 \%\end{array}$ \\
\hline Station de Togba 1 (A3) & $\begin{array}{l}\text { vieille jachère localisée sur } \\
\text { une pente au contact du } \\
\text { marécage de Togba }\end{array}$ & $\begin{array}{l}\text {-Tectona grandis et } \\
\text { Eucalyptus camaldulensis de } \\
17 \text { m de haut } \\
\text {-recouvrement de } 20 \%\end{array}$ & $\begin{array}{l}\text {-Manguifera indica et Citrus sp. de } \\
5 \text { m de haut } \\
\quad \text {-recouvrement de } 30 \%\end{array}$ & $\begin{array}{l}\text {-strate herbacée de sous-bois de } 1 \mathrm{~m} \text { de hauteur } \\
\text { Chromolaena odorata } \\
\text {-recouvrement } 80 \%\end{array}$ \\
\hline Station de Togba 2 (A4) & $\begin{array}{l}\text { une ancienne jachère sous } \\
\text { palmier à huile }\end{array}$ & $\begin{array}{l}\text {-Elaeis guineensis de hauteur } \\
\text { entre } 10 \mathrm{~m} \text { et } 15 \mathrm{~m} \\
\text { - recouvrement de } 20 \%\end{array}$ & $\begin{array}{l}\text {-Chromolaena odorata et jeunes } \\
\text { sujets de Dialium guineense de } 3 \mathrm{~m} \\
\text { de haut }\end{array}$ & Inexistante \\
\hline
\end{tabular}




\section{T. O. LOUGBÉGNON et al. /Int. J. Biol. Chem. Sci. 4(4): 1191-1216, 2010}

\begin{tabular}{|c|c|c|c|c|}
\hline & & & Recouvrement de $100 \%$. & \\
\hline Station de Togba 3 (A5) & $\begin{array}{c}\text { jeune jachère sous Elaeis } \\
\text { guineensis }\end{array}$ & Inexistante & $\begin{array}{l}\text { - Elaeis guineensis de } 5 \mathrm{~m} \text { de haut } \\
\text { et de jeunes pieds de Zanthoxylum } \\
\text { zanthoxyloides, Vitex doniana et } \\
\text { Psidium guajava } \\
\quad \text { - recouvrement de } 60 \%\end{array}$ & $\begin{array}{c}\text { - strate de sous-bois de hauteur } 1 \mathrm{~m} \text { dominée par } \\
\text { Panicum sp. et Andropogon gayanus } \\
\text { - recouvrement de } 50 \%\end{array}$ \\
\hline
\end{tabular}

Tableau 2: Composition de l'avifaune des mosaïques de milieux prospectés ainsi que l'occurrence des espèces dans les stations notée en présence-absence.

\begin{tabular}{|c|c|c|c|c|c|c|c|c|c|c|c|c|c|c|c|c|c|c|c|c|c|c|c|c|c|c|}
\hline \multirow[t]{2}{*}{$\mathbf{N}^{\circ}$} & \multirow{2}{*}{$\begin{array}{l}\text { Nom scientifique et } \\
\text { code }\end{array}$} & \multicolumn{12}{|c|}{ Oiseaux des plantations } & \multicolumn{13}{|c|}{ Oiseaux des jachères } \\
\hline & & $T 1$ & $T 2$ & $T 3$ & $T 4$ & $P 1$ & $P 2$ & $C 1$ & $C 2$ & 01 & 02 & $E 1$ & $E 2$ & $l 1$ & 12 & D1 & D2 & D3 & D4 & $A 1$ & $A 2$ & $A 3$ & $A 4$ & $A 5$ & O3 & 04 \\
\hline & Ardeidae & & & & & & & & & & & & & & & & & & & & & & & & & \\
\hline 1 & Bulbucus ibis (Bui) & 1 & 1 & 1 & 1 & 1 & 1 & 1 & 1 & 1 & 1 & 1 & 1 & 1 & 1 & 1 & 1 & 1 & 1 & 1 & 1 & 1 & 1 & 1 & 1 & 1 \\
\hline 2 & $\begin{array}{l}\text { Butorides striatus } \\
\text { (Bus) }\end{array}$ & 0 & 0 & 0 & 0 & 0 & 0 & 0 & 0 & 0 & 0 & 0 & 0 & 0 & 0 & 0 & 0 & 0 & 0 & 0 & 0 & 1 & 1 & 0 & 0 & 0 \\
\hline & Accipitridae & & & & & & & & & & & & & & & & & & & & & & & & & \\
\hline 3 & $\begin{array}{l}\text { Elanus caeruleus } \\
\text { (Elc) }\end{array}$ & 0 & 1 & 1 & 1 & 1 & 1 & 0 & 1 & 0 & 0 & 1 & 0 & 1 & 0 & 1 & 1 & 1 & 0 & 0 & 1 & 0 & 1 & 1 & 1 & 1 \\
\hline 4 & $\begin{array}{l}\text { Milvus migrans } \\
(\mathbf{M i m})\end{array}$ & 1 & 1 & 1 & 1 & 1 & 1 & 1 & 1 & 1 & 1 & 1 & 1 & 1 & 1 & 1 & 1 & 1 & 1 & 1 & 1 & 1 & 1 & 1 & 1 & 1 \\
\hline 5 & $\begin{array}{l}\text { Gypohierax } \\
\text { angolensis (Gya) }\end{array}$ & 0 & 0 & 0 & 0 & 0 & 0 & 0 & 0 & 0 & 0 & 1 & 1 & 0 & 0 & 0 & 0 & 0 & 0 & 0 & 0 & 0 & 0 & 0 & 0 & 0 \\
\hline 6 & $\begin{array}{l}\text { Polyboroides typus } \\
\text { (Pot) }\end{array}$ & 0 & 0 & 1 & 0 & 0 & 0 & 0 & 0 & 0 & 0 & 0 & 0 & 0 & 0 & 0 & 0 & 0 & 0 & 0 & 0 & 0 & 0 & 0 & 0 & 0 \\
\hline 7 & $\begin{array}{l}\text { Kaupifalco } \\
\text { monogramicus } \\
\text { (Kam) }\end{array}$ & 0 & 1 & 1 & 1 & 1 & 1 & 1 & 1 & 1 & 1 & 1 & 1 & 0 & 1 & 0 & 0 & 0 & 0 & 0 & 0 & 0 & 0 & 0 & 1 & 1 \\
\hline 8 & $\begin{array}{l}\text { Accipiter badius } \\
(\mathbf{A c b})\end{array}$ & 1 & 1 & 1 & 1 & 1 & 1 & 1 & 1 & 1 & 1 & 1 & 1 & 0 & 1 & 0 & 0 & 1 & 1 & 1 & 1 & 0 & 1 & 1 & 1 & 1 \\
\hline & Falconidae & & & & & & & & & & & & & & & & & & & & & & & & & \\
\hline 9 & $\begin{array}{l}\text { Falco ardosiaceus } \\
\text { (Faa) }\end{array}$ & 0 & 0 & 0 & 0 & 0 & 0 & 0 & 0 & 0 & 0 & 0 & 0 & 1 & 1 & 1 & 1 & 0 & 0 & 0 & 0 & 0 & 0 & 0 & 1 & 1 \\
\hline
\end{tabular}


T. O. LOUGBÉGNON et al. / Int. J. Biol. Chem. Sci. 4(4): 1191-1216, 2010

\begin{tabular}{|c|c|c|c|c|c|c|c|c|c|c|c|c|c|c|c|c|c|c|c|c|c|c|c|c|c|c|}
\hline 10 & Falco curvieri (Fac) & 0 & 1 & 1 & 1 & 0 & 0 & 0 & 0 & 0 & 0 & 0 & 0 & 0 & 0 & 0 & 0 & 0 & 0 & 0 & 0 & 0 & 0 & 0 & 0 & 0 \\
\hline \multirow[t]{2}{*}{11} & $\begin{array}{l}\text { Falco biarmicus } \\
\text { (Fab) }\end{array}$ & 1 & 1 & 1 & 1 & 0 & 0 & 0 & 0 & 0 & 0 & 0 & 0 & 1 & 1 & 1 & 0 & 0 & 0 & 0 & 0 & 0 & 1 & 0 & 0 & 0 \\
\hline & Phasianidae & & & & & & & & & & & & & & & & & & & & & & & & & \\
\hline \multirow[t]{2}{*}{12} & $\begin{array}{l}\text { Francolin } \\
\text { bicalcaratus (Frb) }\end{array}$ & 1 & 1 & 1 & 1 & 1 & 1 & 0 & 0 & 0 & 0 & 1 & 1 & 1 & 1 & 1 & 1 & 0 & 1 & 1 & 1 & 1 & 1 & 1 & 1 & 1 \\
\hline & Columbidae & & & & & & & & & & & & & & & & & & & & & & & & & \\
\hline 13 & $\begin{array}{l}\text { Streptopelia } \\
\text { semitorquata }(\mathbf{S t s})\end{array}$ & 0 & 1 & 1 & 1 & 0 & 0 & 1 & 1 & 0 & 0 & 1 & 1 & 0 & 0 & 0 & 0 & 0 & 0 & 1 & 0 & 0 & 1 & 1 & 1 & 1 \\
\hline 14 & $\begin{array}{l}\text { Streptopelia vinacea } \\
(\mathbf{S t v})\end{array}$ & 1 & 1 & 1 & 1 & 1 & 1 & 1 & 1 & 1 & 1 & 0 & 0 & 1 & 0 & 1 & 1 & 0 & 0 & 1 & 1 & 0 & 0 & 1 & 0 & 0 \\
\hline 15 & $\begin{array}{l}\text { Streptopelia } \\
\text { senegalensis }(\text { Ste })\end{array}$ & 1 & 1 & 1 & 1 & 1 & 1 & 1 & 1 & 1 & 1 & 1 & 1 & 1 & 0 & 1 & 1 & 0 & 0 & 1 & 1 & 0 & 0 & 1 & 1 & 0 \\
\hline 16 & $\begin{array}{l}\text { Turtur abyssinicus } \\
\text { (Tub) }\end{array}$ & 0 & 0 & 0 & 0 & 0 & 0 & 0 & 0 & 0 & 0 & 0 & 0 & 0 & 1 & 0 & 0 & 0 & 0 & 0 & 0 & 0 & 1 & 1 & 0 & 0 \\
\hline 17 & Turtur afer (Tua) & 1 & 1 & 1 & 1 & 1 & 1 & 0 & 0 & 0 & 0 & 1 & 1 & 0 & 0 & 0 & 0 & 1 & 0 & 0 & 0 & 0 & 1 & 1 & 0 & 1 \\
\hline 18 & $\begin{array}{l}\text { Turtur tympanistria } \\
\text { (Tut) }\end{array}$ & 0 & 0 & 0 & 0 & 0 & 0 & 1 & 1 & 1 & 1 & 0 & 0 & 1 & 0 & 0 & 0 & 0 & 1 & 0 & 0 & 0 & 0 & 1 & 0 & 1 \\
\hline & Psittacidae & & & & & & & & & & & & & & & & & & & & & & & & & \\
\hline 19 & $\begin{array}{l}\text { Psittacula krameri } \\
\text { (Psk) }\end{array}$ & 0 & 0 & 0 & 0 & 0 & 0 & 1 & 1 & 1 & 1 & 0 & 0 & 0 & 1 & 0 & 1 & 1 & 1 & 1 & 1 & 0 & 0 & 0 & 1 & 1 \\
\hline 20 & $\begin{array}{l}\text { Poicephalus } \\
\text { senegalus (Pos) }\end{array}$ & 0 & 0 & 0 & 0 & 0 & 0 & 0 & 0 & 0 & 0 & 0 & 0 & 0 & 1 & 0 & 0 & 0 & 0 & 0 & 0 & 0 & 0 & 0 & 0 & 0 \\
\hline 21 & Musophagidae & & & & & & & & & & & & & & & & & & & & & & & & & \\
\hline 22 & $\begin{array}{l}\text { Crinifer piscator } \\
\text { (Crp) }\end{array}$ & 1 & 1 & 1 & 1 & 1 & 1 & 1 & 1 & 1 & 1 & 1 & 1 & 1 & 1 & 0 & 1 & 1 & 0 & 0 & 0 & 1 & 1 & 1 & 1 & 1 \\
\hline & Cuculidae & & & & & & & & & & & & & & & & & & & & & & & & & \\
\hline 23 & $\begin{array}{l}\text { Chrysococcyx klass } \\
\text { (Chk) }\end{array}$ & 0 & 1 & 1 & 1 & 0 & 0 & 0 & 0 & 0 & 0 & 0 & 0 & 0 & 0 & 0 & 0 & 0 & 0 & 1 & 0 & 0 & 0 & 0 & 0 & 0 \\
\hline 24 & $\begin{array}{l}\text { Chrysococcyx } \\
\text { cupreus (Chc) }\end{array}$ & 0 & 1 & 1 & 1 & 0 & 0 & 0 & 0 & 0 & 0 & 0 & 0 & 0 & 0 & 0 & 0 & 0 & 0 & 0 & 0 & 0 & 0 & 0 & 0 & 0 \\
\hline 25 & $\begin{array}{l}\text { Chrysococcyx } \\
\text { caprius } \text { (Cha) }\end{array}$ & 1 & 1 & 1 & 1 & 0 & 0 & 1 & 1 & 1 & 1 & 1 & 1 & 0 & 1 & 0 & 0 & 0 & 1 & 1 & 1 & 1 & 0 & 0 & 1 & 1 \\
\hline 26 & $\begin{array}{l}\text { Ceuthmochares } \\
\text { aereus } \text { (Cea) }\end{array}$ & 0 & 0 & 1 & 1 & 1 & 1 & 1 & 1 & 1 & 1 & 1 & 1 & 1 & 0 & 0 & 0 & 1 & 1 & 1 & 1 & 0 & 0 & 1 & 1 & 1 \\
\hline
\end{tabular}


T. O. LOUGBÉGNON et al. /Int. J. Biol. Chem. Sci. 4(4): 1191-1216, 2010

\begin{tabular}{|c|c|c|c|c|c|c|c|c|c|c|c|c|c|c|c|c|c|c|c|c|c|c|c|c|c|c|}
\hline 27 & $\begin{array}{l}\text { Centropus grillii } \\
\text { (Ceg) }\end{array}$ & 0 & 1 & 1 & 1 & 0 & 0 & 1 & 0 & 0 & 0 & 1 & 0 & 0 & 1 & 1 & 1 & 0 & 0 & 1 & 1 & 0 & 1 & 0 & 1 & 0 \\
\hline \multirow[t]{2}{*}{28} & $\begin{array}{l}\text { Centropus } \\
\text { senegalensis }(\mathbf{C e s})\end{array}$ & 1 & 1 & 1 & 1 & 1 & 1 & 1 & 1 & 1 & 1 & 1 & 1 & 1 & 1 & 1 & 1 & 1 & 1 & 1 & 1 & 1 & 1 & 1 & 1 & 1 \\
\hline & Tytonidae & & & & & & & & & & & & & & & & & & & & & & & & & \\
\hline \multirow[t]{2}{*}{29} & Tyto alba (Tya) & 0 & 0 & 1 & 1 & 0 & 0 & 0 & 0 & 0 & 0 & 0 & 0 & 0 & 0 & 0 & 0 & 0 & 0 & 1 & 0 & 0 & 0 & 0 & 0 & 0 \\
\hline & Strigidae & & & & & & & & & & & & & & & & & & & & & & & & & \\
\hline 30 & Otus leucotis $($ Otl) & 1 & 1 & 0 & 1 & 0 & 0 & 0 & 0 & 0 & 0 & 0 & 0 & 1 & 1 & 0 & 0 & 0 & 0 & 0 & 0 & 0 & 0 & 1 & 0 & 1 \\
\hline \multirow[t]{2}{*}{31} & $\begin{array}{l}\text { Glaucidium perlatum } \\
\text { (Glp) }\end{array}$ & 0 & 0 & 1 & 1 & 0 & 0 & 0 & 0 & 0 & 0 & 0 & 0 & 0 & 0 & 0 & 0 & 0 & 0 & 0 & 0 & 0 & 0 & 0 & 0 & 0 \\
\hline & Caprimulgidae & & & & & & & & & & & & & & & & & & & & & & & & & \\
\hline 32 & $\begin{array}{l}\text { Caprimulgus } \\
\text { inormatus (Cai) }\end{array}$ & 0 & 0 & 0 & 0 & 1 & 1 & 0 & 0 & 0 & 0 & 1 & 1 & 1 & 1 & 1 & 1 & 0 & 0 & 0 & 1 & 0 & 1 & 0 & 1 & 1 \\
\hline 33 & $\begin{array}{l}\text { Caprimulgus } \\
\text { climacurus }(\mathbf{C a l})\end{array}$ & 1 & 1 & 1 & 1 & 0 & 0 & 0 & 0 & 0 & 0 & 0 & 0 & 1 & 1 & 1 & 1 & 0 & 0 & 0 & 1 & 0 & 0 & 1 & 0 & 1 \\
\hline \multirow[t]{2}{*}{34} & $\begin{array}{l}\text { Macrodipterix } \\
\text { longipennis }(\mathbf{M a l}) \\
\end{array}$ & 1 & 1 & 1 & 1 & 1 & 1 & 1 & 1 & 1 & 1 & 1 & 1 & 1 & 1 & 1 & 1 & 0 & 0 & 0 & 0 & 0 & 1 & 0 & 1 & 1 \\
\hline & Apodidae & & & & & & & & & & & & & & & & & & & & & & & & & \\
\hline 35 & $\begin{array}{l}\text { Cypsiurus parvus } \\
\text { (Cyp) }\end{array}$ & 1 & 1 & 1 & 1 & 1 & 1 & 1 & 1 & 1 & 1 & 1 & 1 & 1 & 1 & 1 & 1 & 0 & 0 & 0 & 0 & 1 & 1 & 1 & 1 & 1 \\
\hline 36 & Apus apus (Apa) & 1 & 1 & 1 & 1 & 1 & 1 & 1 & 1 & 1 & 1 & 1 & 1 & 1 & 1 & 0 & 1 & 1 & 1 & 0 & 1 & 1 & 0 & 0 & 1 & 0 \\
\hline \multirow[t]{2}{*}{37} & Apus affinis (Apf) & 1 & 1 & 1 & 1 & 1 & 1 & 1 & 1 & 1 & 1 & 1 & 1 & 0 & 1 & 1 & 1 & 1 & 1 & 1 & 1 & 1 & 1 & 1 & 1 & 1 \\
\hline & Alcedinidae & & & & & & & & & & & & & & & & & & & & & & & & & \\
\hline 38 & $\begin{array}{l}\text { Alcedo leucogaster } \\
\text { (All) }\end{array}$ & 0 & 0 & 0 & 0 & 0 & 0 & 1 & 1 & 1 & 1 & 0 & 0 & 0 & 0 & 0 & 0 & 0 & 0 & 0 & 0 & 0 & 0 & 0 & 0 & 0 \\
\hline 39 & Ispidina picta (Isp) & 1 & 1 & 1 & 1 & 0 & 0 & 1 & 1 & 1 & 1 & 0 & 0 & 0 & 0 & 0 & 0 & 0 & 1 & 1 & 0 & 0 & 1 & 0 & 1 & 1 \\
\hline 40 & $\begin{array}{l}\text { Halcyon } \\
\text { senegalensis (Has) }\end{array}$ & 1 & 1 & 1 & 1 & 0 & 0 & 1 & 1 & 1 & 1 & 1 & 1 & 0 & 0 & 1 & 1 & 1 & 0 & 1 & 1 & 0 & 1 & 0 & 1 & 0 \\
\hline \multirow[t]{2}{*}{41} & $\begin{array}{l}\text { Halcyon malimbica } \\
\text { (Ham) }\end{array}$ & 1 & 1 & 1 & 1 & 0 & 0 & 1 & 1 & 1 & 1 & 0 & 0 & 0 & 0 & 0 & 0 & 0 & 0 & 0 & 0 & 0 & 0 & 0 & 0 & 0 \\
\hline & Meropidae & & & & & & & & & & & & & & & & & & & & & & & & & \\
\hline 42 & $\begin{array}{l}\text { Merops albicollis } \\
\text { (Mea) }\end{array}$ & 1 & 1 & 1 & 1 & 1 & 1 & 1 & 1 & 1 & 1 & 1 & 1 & 0 & 1 & 1 & 1 & 1 & 1 & 0 & 0 & 1 & 1 & 1 & 1 & 0 \\
\hline 43 & $\begin{array}{l}\text { Merops malimbicus } \\
\text { (Mem) }\end{array}$ & 1 & 1 & 1 & 1 & 0 & 0 & 0 & 0 & 0 & 0 & 0 & 0 & 0 & 1 & 0 & 0 & 0 & 1 & 0 & 0 & 0 & 0 & 0 & 1 & 1 \\
\hline
\end{tabular}




\section{T. O. LOUGBÉGNON et al. /Int. J. Biol. Chem. Sci. 4(4): 1191-1216, 2010}

\begin{tabular}{|c|c|c|c|c|c|c|c|c|c|c|c|c|c|c|c|c|c|c|c|c|c|c|c|c|c|c|}
\hline & Coraciidae & & & & & & & & & & & & & & & & & & & & & & & & & \\
\hline 44 & $\begin{array}{l}\text { Coracias } \\
\text { cyanogaster }(\mathbf{C o c})\end{array}$ & 0 & 0 & 0 & 0 & 0 & 0 & 0 & 0 & 0 & 0 & 0 & 0 & 0 & 1 & 0 & 0 & 0 & 0 & 0 & 0 & 0 & 0 & 0 & 0 & 0 \\
\hline \multirow[t]{2}{*}{45} & $\begin{array}{l}\text { Eurystomus } \\
\text { glaucurus (Eug) }\end{array}$ & 1 & 1 & 1 & 1 & 0 & 0 & 0 & 0 & 0 & 0 & 0 & 0 & 0 & 0 & 0 & 0 & 0 & 0 & 0 & 0 & 0 & 0 & 0 & 0 & 0 \\
\hline & Phoeniculidae & & & & & & & & & & & & & & & & & & & & & & & & & \\
\hline \multirow[t]{2}{*}{46} & $\begin{array}{l}\text { Phoeniculus } \\
\text { purpureus (Php) }\end{array}$ & 1 & 1 & 1 & 1 & 1 & 1 & 1 & 1 & 1 & 1 & 1 & 1 & 0 & 0 & 0 & 0 & 0 & 0 & 0 & 0 & 0 & 0 & 0 & 0 & 0 \\
\hline & Bucerotidae & & & & & & & & & & & & & & & & & & & & & & & & & \\
\hline \multirow[t]{2}{*}{47} & Tockus nasitus (Ton) & 0 & 0 & 0 & 0 & 1 & 1 & 1 & 1 & 1 & 1 & 1 & 1 & 1 & 1 & 0 & 0 & 0 & 0 & 0 & 0 & 0 & 0 & 0 & 0 & 0 \\
\hline & Capitonidae & & & & & & & & & & & & & & & & & & & & & & & & & \\
\hline 48 & $\begin{array}{l}\text { Pogoniulus } \\
\text { bilineatus }(\mathbf{P o b})\end{array}$ & 0 & 1 & 1 & 1 & 0 & 0 & 0 & 0 & 0 & 0 & 0 & 0 & 0 & 0 & 0 & 0 & 0 & 0 & 0 & 0 & 0 & 0 & 0 & 0 & 0 \\
\hline \multirow[t]{2}{*}{49} & $\begin{array}{l}\text { Lybius bidentatus } \\
\text { (Lyb) }\end{array}$ & 1 & 1 & 1 & 1 & 0 & 0 & 1 & 1 & 1 & 1 & 0 & 0 & 0 & 0 & 0 & 0 & 0 & 0 & 0 & 0 & 0 & 0 & 0 & 0 & 0 \\
\hline & Picidae & & & & & & & & & & & & & & & & & & & & & & & & & \\
\hline 50 & $\begin{array}{l}\text { Campethera } \\
\text { caillautii }(\mathbf{C a m})\end{array}$ & 0 & 0 & 1 & 1 & 0 & 0 & 0 & 0 & 0 & 0 & 0 & 0 & 0 & 0 & 0 & 0 & 1 & 1 & 0 & 0 & 0 & 1 & 0 & 0 & 0 \\
\hline 51 & $\begin{array}{l}\text { Dendropicos } \\
\text { pyrrhogaster }(\text { Dep) }\end{array}$ & 0 & 0 & 0 & 0 & 1 & 1 & 1 & 1 & 1 & 1 & 1 & 1 & 0 & 0 & 0 & 0 & 0 & 0 & 0 & 0 & 0 & 1 & 1 & 0 & 0 \\
\hline \multirow[t]{2}{*}{52} & $\begin{array}{l}\text { Dendropicos goertae } \\
\text { (Deg) }\end{array}$ & 1 & 1 & 1 & 1 & 1 & 1 & 1 & 1 & 1 & 1 & 1 & 1 & 1 & 1 & 1 & 1 & 1 & 1 & 1 & 1 & 1 & 1 & 1 & 1 & 1 \\
\hline & Eurylaimidae & & & & & & & & & & & & & & & & & & & & & & & & & \\
\hline \multirow[t]{7}{*}{53} & $\begin{array}{l}\text { Smithomis capensis } \\
(\mathbf{S m c})\end{array}$ & 0 & 0 & 0 & 0 & 0 & 0 & 0 & 0 & 0 & 0 & 0 & 0 & 0 & 1 & 0 & 0 & 0 & 0 & 0 & 0 & 0 & 0 & 0 & 0 & 0 \\
\hline & Hirundinidae & & & & & & & & & & & & & & & & & & & & & & & & & \\
\hline & $\begin{array}{l}\text { Hirundo rustica } \\
\text { (Hir) }\end{array}$ & 1 & 1 & 1 & 1 & 1 & 1 & 1 & 1 & 1 & 1 & 1 & 1 & 1 & 1 & 1 & 1 & 0 & 0 & 0 & 1 & 1 & 0 & 0 & 0 & 0 \\
\hline & $\begin{array}{l}\text { Hirundo smithii } \\
\text { (His) }\end{array}$ & 0 & 0 & 0 & 0 & 1 & 1 & 1 & 1 & 1 & 1 & 1 & 1 & 0 & 1 & 0 & 0 & 0 & 1 & 0 & 0 & 0 & 1 & 1 & 0 & 0 \\
\hline & $\begin{array}{l}\text { Hirundo semirufa } \\
\text { (Hie) }\end{array}$ & 1 & 1 & 1 & 1 & 1 & 1 & 1 & 1 & 1 & 1 & 1 & 1 & 1 & 1 & 1 & 1 & 1 & 1 & 1 & 1 & 1 & 1 & 1 & 1 & 1 \\
\hline & $\begin{array}{l}\text { Hirundo } \\
\text { senegalensis (Hin) }\end{array}$ & 1 & 1 & 1 & 1 & 1 & 1 & 1 & 1 & 1 & 1 & 1 & 1 & 1 & 1 & 1 & 1 & 1 & 1 & 1 & 1 & 1 & 1 & 1 & 1 & 1 \\
\hline & Hirundo abyssinica & 1 & 1 & 1 & 1 & 1 & 1 & 1 & 1 & 1 & 1 & 1 & 1 & 1 & 1 & 0 & 1 & 1 & 0 & 0 & 1 & 0 & 1 & 1 & 0 & 0 \\
\hline
\end{tabular}




\section{T. O. LOUGBÉGNON et al. /Int. J. Biol. Chem. Sci. 4(4): 1191-1216, 2010}

\begin{tabular}{|c|c|c|c|c|c|c|c|c|c|c|c|c|c|c|c|c|c|c|c|c|c|c|c|c|c|}
\hline (Hia) & & & & & & & & & & & & & & & & & & & & & & & & & \\
\hline \multicolumn{26}{|l|}{ Motacillidae } \\
\hline $\begin{array}{l}\text { Anthus leucophrys } \\
\text { (Anl) }\end{array}$ & 1 & 1 & 1 & 1 & 0 & 0 & 0 & 0 & 0 & 0 & 0 & 0 & 1 & 1 & 0 & 0 & 0 & 0 & 0 & 0 & 0 & 0 & 0 & 0 & 0 \\
\hline $\begin{array}{l}\text { Macronyx croceus } \\
\text { (Mac) }\end{array}$ & 0 & 0 & 0 & 0 & 0 & 0 & 1 & 1 & 0 & 1 & 0 & 0 & 0 & 0 & 0 & 0 & 1 & 1 & 0 & 0 & 0 & 0 & 0 & 0 & 0 \\
\hline $\begin{array}{l}\text { Motacilla flava } \\
\text { (Mof) }\end{array}$ & 1 & 1 & 1 & 1 & 0 & 0 & 1 & 1 & 1 & 1 & 0 & 0 & 1 & 1 & 1 & 1 & 1 & 0 & 0 & 1 & 0 & 1 & 1 & 1 & 1 \\
\hline \multicolumn{26}{|l|}{ Pycnonotidae } \\
\hline $\begin{array}{l}\text { Pycnonotus barbatus } \\
\text { (Pyb) }\end{array}$ & 1 & 1 & 1 & 1 & 1 & 1 & 1 & 1 & 1 & 1 & 1 & 1 & 1 & 1 & 1 & 1 & 1 & 1 & 1 & 1 & 1 & 1 & 1 & 1 & 1 \\
\hline $\begin{array}{l}\text { Andropadus virens } \\
\text { (Anv) }\end{array}$ & 0 & 0 & 0 & 0 & 0 & 0 & 1 & 1 & 0 & 0 & 0 & 0 & 0 & 0 & 0 & 0 & 0 & 0 & 0 & 0 & 0 & 0 & 0 & 0 & 0 \\
\hline $\begin{array}{l}\text { Chlorocichla simplex } \\
\text { (Chs) }\end{array}$ & 0 & 0 & 0 & 0 & 0 & 0 & 1 & 1 & 0 & 0 & 0 & 0 & 0 & 0 & 0 & 0 & 0 & 1 & 0 & 0 & 0 & 0 & 1 & 1 & 1 \\
\hline $\begin{array}{l}\text { Chlorocichla } \\
\text { flavicollis }(\mathbf{C h f})\end{array}$ & 0 & 1 & 1 & 0 & 0 & 0 & 0 & 0 & 0 & 0 & 0 & 0 & 0 & 0 & 0 & 0 & 0 & 0 & 0 & 0 & 0 & 0 & 0 & 0 & 0 \\
\hline $\begin{array}{l}\text { Thescelocichla } \\
\text { leucopleura (Ths) }\end{array}$ & 0 & 0 & 0 & 0 & 0 & 0 & 1 & 1 & 0 & 0 & 0 & 0 & 0 & 0 & 0 & 0 & 0 & 0 & 0 & 0 & 1 & 0 & 0 & 0 & 0 \\
\hline Nicator chloris (Nic) & 0 & 0 & 0 & 0 & 0 & 0 & 0 & 0 & 0 & 0 & 0 & 0 & 0 & 0 & 0 & 0 & 0 & 0 & 0 & 0 & 0 & 0 & 0 & 1 & 1 \\
\hline \multicolumn{26}{|l|}{ Turdidae } \\
\hline $\begin{array}{l}\text { Saxicola rubetra } \\
\text { (Sar) }\end{array}$ & 1 & 1 & 0 & 0 & 0 & 0 & 0 & 0 & 0 & 0 & 0 & 0 & 0 & 1 & 0 & 0 & 0 & 0 & 0 & 0 & 0 & 0 & 0 & 0 & 0 \\
\hline Turdus pelios (Tup) & 1 & 1 & 1 & 1 & 1 & 1 & 1 & 1 & 1 & 1 & 1 & 1 & 1 & 1 & 1 & 1 & 1 & 1 & 1 & 1 & 1 & 1 & 1 & 1 & 1 \\
\hline \multicolumn{26}{|l|}{ Sylviidae } \\
\hline $\begin{array}{l}\text { Hippolais pallida } \\
\text { (Hyp) }\end{array}$ & 1 & 0 & 0 & 0 & 1 & 1 & 1 & 1 & 0 & 0 & 0 & 0 & 1 & 1 & 1 & 1 & 1 & 0 & 0 & 0 & 1 & 1 & 0 & 1 & 1 \\
\hline $\begin{array}{l}\text { Cisticola anonymus } \\
\text { (Cin) }\end{array}$ & 1 & 1 & 1 & 1 & 0 & 0 & 1 & 1 & 1 & 1 & 0 & 0 & 0 & 0 & 0 & 0 & 0 & 0 & 0 & 0 & 0 & 0 & 0 & 0 & 0 \\
\hline $\begin{array}{l}\text { Cisticola } \\
\text { brachypterus (Cib) }\end{array}$ & 1 & 1 & 1 & 1 & 0 & 0 & 1 & 1 & 1 & 1 & 0 & 0 & 1 & 1 & 1 & 1 & 0 & 0 & 0 & 0 & 0 & 0 & 0 & 1 & 0 \\
\hline Prinia subflava (Prs) & 1 & 1 & 1 & 1 & 0 & 0 & 0 & 0 & 0 & 0 & 0 & 0 & 0 & 1 & 0 & 1 & 1 & 0 & 0 & 0 & 0 & 0 & 0 & 0 & 0 \\
\hline $\begin{array}{l}\text { Camaroptera } \\
\text { brachyura }(\mathbf{C a b})\end{array}$ & 1 & 1 & 1 & 1 & 1 & 1 & 1 & 1 & 1 & 1 & 1 & 1 & 1 & 1 & 1 & 1 & 0 & 0 & 1 & 1 & 0 & 0 & 1 & 1 & 1 \\
\hline Phylloscopus & 1 & 1 & 1 & 1 & 0 & 0 & 1 & 1 & 1 & 1 & 0 & 0 & 0 & 1 & 0 & 0 & 0 & 1 & 0 & 0 & 0 & 0 & 0 & 0 & 0 \\
\hline
\end{tabular}


T. O. LOUGBÉGNON et al. / Int. J. Biol. Chem. Sci. 4(4): 1191-1216, 2010

\begin{tabular}{|c|c|c|c|c|c|c|c|c|c|c|c|c|c|c|c|c|c|c|c|c|c|c|c|c|c|}
\hline trochillus (Pht) & & & & & & & & & & & & & & & & & & & & & & & & & \\
\hline $\begin{array}{l}\text { Sylvietta virens } \\
(\mathbf{S y v})\end{array}$ & 1 & 1 & 1 & 1 & 0 & 0 & 0 & 0 & 0 & 0 & 0 & 0 & 0 & 0 & 0 & 0 & 0 & 0 & 0 & 0 & 0 & 0 & 0 & 0 & 0 \\
\hline Sylvia Borin $(\mathbf{S y b})$ & 1 & 1 & 1 & 1 & 1 & 1 & 1 & 1 & 1 & 1 & 1 & 1 & 1 & 1 & 0 & 1 & 1 & 0 & 0 & 0 & 0 & 1 & 1 & 0 & 0 \\
\hline \multicolumn{26}{|l|}{ Muscicapidae } \\
\hline $\begin{array}{l}\text { Muscicapa striata } \\
\text { (Mus) }\end{array}$ & 1 & 1 & 1 & 1 & 0 & 0 & 0 & 0 & 0 & 0 & 0 & 0 & 0 & 1 & 0 & 0 & 0 & 0 & 0 & 0 & 0 & 0 & 0 & 0 & 0 \\
\hline $\begin{array}{l}\text { Myioparus plumbeus } \\
\text { (Myp) }\end{array}$ & 1 & 1 & 0 & 0 & 0 & 0 & 0 & 0 & 0 & 0 & 0 & 0 & 1 & 0 & 0 & 0 & 0 & 0 & 0 & 0 & 0 & 0 & 1 & 0 & 0 \\
\hline \multicolumn{26}{|l|}{ Timaliidae } \\
\hline $\begin{array}{l}\text { Tudoides plebejus } \\
\text { (Tul) }\end{array}$ & 1 & 1 & 1 & 1 & 1 & 1 & 1 & 1 & 1 & 1 & 1 & 1 & 1 & 1 & 1 & 1 & 1 & 1 & 1 & 1 & 1 & 1 & 1 & 1 & 1 \\
\hline \multicolumn{26}{|l|}{ Nectariniidae } \\
\hline $\begin{array}{l}\text { Anthreptes } \\
\text { gabonicus (Anb) }\end{array}$ & 0 & 0 & 0 & 0 & 0 & 0 & 1 & 1 & 1 & 1 & 0 & 0 & 0 & 0 & 0 & 0 & 0 & 0 & 0 & 0 & 0 & 0 & 0 & 0 & 0 \\
\hline $\begin{array}{l}\text { Anthreptes collaris } \\
\text { (Anc) }\end{array}$ & 0 & 0 & 0 & 0 & 1 & 1 & 1 & 1 & 1 & 1 & 1 & 1 & 0 & 0 & 0 & 1 & 1 & 1 & 1 & 1 & 0 & 0 & 1 & 1 & 1 \\
\hline $\begin{array}{l}\text { Cyanomitra obscura } \\
\text { (Cуо) }\end{array}$ & 1 & 1 & 1 & 1 & 1 & 1 & 1 & 1 & 1 & 1 & 1 & 1 & 0 & 0 & 0 & 1 & 1 & 1 & 1 & 1 & 0 & 1 & 1 & 1 & 1 \\
\hline $\begin{array}{l}\text { Chalcomitra } \\
\text { senegalensis }(\text { Che) }\end{array}$ & 1 & 1 & 1 & 1 & 1 & 1 & 1 & 1 & 1 & 1 & 1 & 1 & 1 & 1 & 1 & 1 & 0 & 0 & 0 & 0 & 0 & 0 & 0 & 1 & 1 \\
\hline $\begin{array}{l}\text { Cinnyris } \\
\text { chloropygius (Cic) }\end{array}$ & 1 & 1 & 1 & 1 & 0 & 0 & 0 & 0 & 0 & 0 & 0 & 0 & 1 & 1 & 0 & 0 & 0 & 0 & 0 & 0 & 0 & 0 & 0 & 0 & 0 \\
\hline $\begin{array}{l}\text { Cinnyris } \\
\text { coccinigaster (Cio) }\end{array}$ & 1 & 1 & 1 & 1 & 1 & 1 & 1 & 1 & 1 & 1 & 1 & 1 & 1 & 1 & 1 & 1 & 0 & 0 & 0 & 0 & 0 & 0 & 0 & 0 & 0 \\
\hline $\begin{array}{l}\text { Cinnyris venustus } \\
\text { (Civ) }\end{array}$ & 1 & 1 & 1 & 1 & 1 & 1 & 1 & 1 & 1 & 1 & 1 & 1 & 1 & 1 & 1 & 1 & 0 & 0 & 0 & 0 & 0 & 0 & 0 & 0 & 1 \\
\hline $\begin{array}{l}\text { Cinnyris cupreus } \\
\text { (Ciu) }\end{array}$ & 1 & 1 & 1 & 1 & 1 & 1 & 1 & 1 & 1 & 1 & 1 & 1 & 1 & 1 & 0 & 0 & 1 & 0 & 0 & 0 & 0 & 0 & 0 & 0 & 0 \\
\hline \multicolumn{26}{|l|}{ Laniidae } \\
\hline $\begin{array}{l}\text { Corvinella corvina } \\
\text { (Cor) }\end{array}$ & 1 & 1 & 1 & 1 & 1 & 1 & 1 & 1 & 1 & 1 & 1 & 1 & 1 & 1 & 1 & 1 & 1 & 1 & 1 & 1 & 1 & 1 & 1 & 1 & 1 \\
\hline \multicolumn{26}{|l|}{ Malaconotidae } \\
\hline $\begin{array}{l}\text { Tchagra senegala } \\
\text { (Tcs) }\end{array}$ & 1 & 1 & 1 & 1 & 1 & 1 & 1 & 1 & 1 & 1 & 1 & 1 & 1 & 1 & 1 & 1 & 1 & 1 & 1 & 1 & 1 & 1 & 1 & 1 & 1 \\
\hline
\end{tabular}




\section{T. O. LOUGBÉGNON et al. /Int. J. Biol. Chem. Sci. 4(4): 1191-1216, 2010}

\begin{tabular}{|c|c|c|c|c|c|c|c|c|c|c|c|c|c|c|c|c|c|c|c|c|c|c|c|c|c|}
\hline Prionopidae & & & & & & & & & & & & & & & & & & & & & & & & & \\
\hline $\begin{array}{l}\text { Prionops plumatus } \\
\text { (Prp) }\end{array}$ & 1 & 1 & 1 & 1 & 1 & 1 & 1 & 1 & 1 & 1 & 1 & 1 & 0 & 0 & 1 & 1 & 0 & 0 & 0 & 0 & 0 & 1 & 1 & 0 & 0 \\
\hline \multicolumn{26}{|l|}{ Dicruridae } \\
\hline $\begin{array}{l}\text { Dicrurus adsimilis } \\
\text { (Dia) }\end{array}$ & 1 & 1 & 1 & 1 & 1 & 1 & 1 & 1 & 1 & 1 & 1 & 1 & 1 & 0 & 0 & 0 & 0 & 1 & 0 & 0 & 0 & 1 & 1 & 1 & 1 \\
\hline \multicolumn{26}{|l|}{ Corvidae } \\
\hline Corvus albus (Coa) & 1 & 1 & 1 & 1 & 1 & 1 & 1 & 1 & 1 & 1 & 1 & 1 & 0 & 0 & 1 & 1 & 0 & 0 & 1 & 1 & 0 & 0 & 0 & 0 & 0 \\
\hline $\begin{array}{l}\begin{array}{l}\text { Ptilostomus afer } \\
\text { (Pta) }\end{array} \\
\end{array}$ & 0 & 0 & 0 & 0 & 0 & 0 & 1 & 1 & 1 & 1 & 0 & 0 & 0 & 0 & 0 & 0 & 0 & 0 & 1 & 1 & 0 & 0 & 0 & 0 & 0 \\
\hline \multicolumn{26}{|l|}{ Sturnidae } \\
\hline $\begin{array}{l}\text { Lamprotornis } \\
\text { purpureus (Lap) }\end{array}$ & 0 & 0 & 0 & 0 & 0 & 0 & 1 & 1 & 1 & 1 & 0 & 0 & 0 & 0 & 0 & 0 & 0 & 0 & 0 & 0 & 0 & 0 & 0 & 0 & 0 \\
\hline $\begin{array}{l}\text { Lamprotornis } \\
\text { splendidus } \text { (Las) }\end{array}$ & 0 & 0 & 0 & 0 & 0 & 0 & 0 & 0 & 0 & 0 & 0 & 0 & 0 & 0 & 0 & 0 & 0 & 0 & 1 & 0 & 0 & 0 & 0 & 0 & 0 \\
\hline \multicolumn{26}{|l|}{ Passeridae } \\
\hline $\begin{array}{l}\text { Passer domesticus } \\
\text { (Pad) }\end{array}$ & 0 & 0 & 0 & 0 & 0 & 0 & 1 & 1 & 1 & 1 & 0 & 0 & 0 & 0 & 1 & 1 & 0 & 0 & 0 & 1 & 0 & 1 & 0 & 0 & 0 \\
\hline Passer griseus $(\mathbf{P a g})$ & 1 & 1 & 1 & 1 & 1 & 1 & 1 & 1 & 1 & 1 & 0 & 0 & 1 & 1 & 1 & 1 & 0 & 0 & 0 & 1 & 0 & 0 & 0 & 0 & 0 \\
\hline $\begin{array}{l}\text { Petronia dentata } \\
\text { (Ped) }\end{array}$ & 1 & 1 & 1 & 1 & 1 & 1 & 1 & 1 & 1 & 1 & 1 & 1 & 0 & 0 & 1 & 1 & 1 & 1 & 0 & 1 & 1 & 1 & 1 & 1 & 1 \\
\hline \multicolumn{26}{|l|}{ Ploceidae } \\
\hline $\begin{array}{l}\text { Plocepasser } \\
\text { superciliosus (PIs) }\end{array}$ & 1 & 1 & 1 & 1 & 1 & 1 & 1 & 1 & 1 & 1 & 1 & 1 & 1 & 1 & 1 & 1 & 0 & 0 & 0 & 1 & 1 & 1 & 0 & 1 & 1 \\
\hline $\begin{array}{l}\text { Ploceus aurantius } \\
(\mathbf{P l u})\end{array}$ & 0 & 0 & 0 & 0 & 0 & 0 & 1 & 1 & 1 & 0 & 0 & 0 & 0 & 0 & 0 & 0 & 0 & 0 & 0 & 0 & 0 & 0 & 0 & 0 & 0 \\
\hline $\begin{array}{l}\text { Ploceus nigerrimus } \\
(\mathbf{P l i})\end{array}$ & 1 & 1 & 1 & 1 & 1 & 1 & 1 & 1 & 1 & 1 & 1 & 1 & 1 & 1 & 1 & 1 & 1 & 1 & 1 & 1 & 1 & 1 & 1 & 1 & 1 \\
\hline $\begin{array}{l}\text { Ploceus cucullatus } \\
\text { (Plm) }\end{array}$ & 1 & 1 & 1 & 1 & 1 & 1 & 1 & 1 & 1 & 1 & 1 & 1 & 1 & 1 & 1 & 1 & 1 & 1 & 1 & 1 & 1 & 1 & 1 & 1 & 1 \\
\hline $\begin{array}{l}\text { Ploceus } \\
\text { melanocephalus } \\
\text { (Plm) }\end{array}$ & 0 & 0 & 0 & 0 & 0 & 0 & 0 & 0 & 0 & 0 & 0 & 0 & 0 & 0 & 0 & 0 & 1 & 0 & 0 & 0 & 0 & 1 & 1 & 1 & 1 \\
\hline $\begin{array}{l}\text { Ploceus } \\
\text { superciliosus (Plp) }\end{array}$ & 1 & 1 & 1 & 1 & 0 & 0 & 1 & 1 & 1 & 1 & 0 & 0 & 0 & 0 & 1 & 1 & 0 & 0 & 0 & 1 & 0 & 1 & 1 & 1 & 1 \\
\hline
\end{tabular}


T. O. LOUGBÉGNON et al. /Int. J. Biol. Chem. Sci. 4(4): 1191-1216, 2010

\begin{tabular}{|c|c|c|c|c|c|c|c|c|c|c|c|c|c|c|c|c|c|c|c|c|c|c|c|c|c|}
\hline $\begin{array}{l}\text { Malimbus scutatus } \\
\text { (Mas) }\end{array}$ & 1 & 1 & 1 & 1 & 1 & 1 & 0 & 0 & 0 & 0 & 1 & 1 & 0 & 1 & 0 & 1 & 1 & 0 & 0 & 0 & 0 & 1 & 1 & 1 & 1 \\
\hline $\begin{array}{l}\text { Quelea erythrops } \\
\text { (Que) }\end{array}$ & 0 & 0 & 0 & 0 & 1 & 1 & 0 & 0 & 0 & 0 & 1 & 1 & 0 & 1 & 0 & 0 & 1 & 1 & 0 & 0 & 0 & 0 & 0 & 1 & 1 \\
\hline $\begin{array}{l}\text { Euplectes } \\
\text { hordeaceus (Euh) }\end{array}$ & 1 & 1 & 1 & 1 & 1 & 1 & 1 & 1 & 1 & 1 & 1 & 1 & 0 & 1 & 1 & 1 & 1 & 0 & 0 & 1 & 1 & 0 & 0 & 0 & 0 \\
\hline $\begin{array}{l}\text { Euplectes } \\
\text { franciscanus (Euf) }\end{array}$ & 1 & 1 & 1 & 1 & 1 & 1 & 0 & 0 & 0 & 0 & 1 & 1 & 1 & 1 & 1 & 1 & 0 & 0 & 0 & 1 & 1 & 0 & 0 & 1 & 1 \\
\hline $\begin{array}{l}\text { Euplectes macrourus } \\
\text { (Eum) }\end{array}$ & 1 & 1 & 1 & 1 & 1 & 1 & 1 & 1 & 1 & 1 & 1 & 1 & 1 & 1 & 1 & 1 & 0 & 0 & 0 & 1 & 1 & 0 & 0 & 1 & 1 \\
\hline $\begin{array}{l}\text { Estrildidae } \\
\begin{array}{l}\text { Nigrita canicapilla } \\
\text { (Nia) }\end{array}\end{array}$ & 0 & 1 & 0 & 1 & 1 & 1 & 1 & 1 & 1 & 1 & 1 & 1 & 0 & 0 & 0 & 1 & 1 & 0 & 0 & 1 & 0 & 1 & 1 & 1 & 0 \\
\hline $\begin{array}{l}\text { Lagonosticta } \\
\text { senegala }(\text { Lae) }\end{array}$ & 1 & 1 & 1 & 1 & 1 & 1 & 1 & 1 & 1 & 1 & 1 & 1 & 1 & 1 & 1 & 1 & 0 & 0 & 0 & 1 & 1 & 0 & 0 & 0 & 0 \\
\hline $\begin{array}{l}\text { Estrilda melpoda } \\
(\mathbf{E s m})\end{array}$ & 1 & 1 & 1 & 1 & 1 & 1 & 0 & 0 & 0 & 0 & 1 & 1 & 1 & 0 & 1 & 1 & 0 & 0 & 0 & 1 & 0 & 0 & 0 & 0 & 1 \\
\hline $\begin{array}{l}\text { Uraeginthus } \\
\text { bengalus (Urb) }\end{array}$ & 1 & 1 & 1 & 1 & 1 & 1 & 1 & 1 & 1 & 1 & 1 & 1 & 0 & 1 & 1 & 1 & 0 & 0 & 0 & 1 & 0 & 0 & 0 & 0 & 0 \\
\hline $\begin{array}{l}\text { Lonchura cucullata } \\
\text { (Loc) }\end{array}$ & 1 & 1 & 1 & 1 & 1 & 1 & 1 & 1 & 1 & 1 & 1 & 1 & 1 & 1 & 1 & 1 & 1 & 1 & 0 & 1 & 1 & 1 & 0 & 1 & 1 \\
\hline $\begin{array}{l}\text { Lonchura bicolour } \\
\text { (Lob) }\end{array}$ & 1 & 1 & 1 & 1 & 1 & 1 & 1 & 1 & 1 & 1 & 1 & 1 & 1 & 0 & 0 & 0 & 1 & 1 & 0 & 1 & 1 & 0 & 0 & 0 & 0 \\
\hline Viduidae & & & & & & & & & & & & & & & & & & & & & & & & & \\
\hline $\begin{array}{l}\text { Vidua chalybeata } \\
\text { (Vic) }\end{array}$ & 1 & 1 & 1 & 1 & 0 & 0 & 0 & 0 & 0 & 0 & 0 & 0 & 0 & 1 & 1 & 1 & 0 & 0 & 0 & 1 & 0 & 0 & 0 & 0 & 1 \\
\hline $\begin{array}{l}\text { Vidua macroura } \\
\text { (Vim) }\end{array}$ & 1 & 1 & 1 & 1 & 1 & 1 & 0 & 0 & 0 & 0 & 0 & 0 & 0 & 1 & 1 & 1 & 0 & 0 & 1 & 1 & 1 & 0 & 0 & 0 & 0 \\
\hline
\end{tabular}


(Las) qui sont très exigeantes de l'existence de végétation ligneuse. Elles peuvent être assimilables au groupe 3 ;

- les espèces rencontrées dans deux stations expriment plusieurs réalités. En effet, on retrouve ici les oiseaux des plantations très boisées comme Gypohierax angolensis (Gya), Glaucidium perlatum (Glp) et, des espèces de jachères buissonnantes ou de sous-bois buissonnants d'Elaeis guinnensis [Andropadus virens (Anv), Chlorocichla flavicollis (Chf), Nicator chloris (Nic)] et même une espèce très accidentelle des milieux humides ou marécageux: Butorides striatus (Bus). Ce groupe est donc assimilable au groupe 2 des oiseaux ubiquistes entre plantations et jachères.

- les espèces apparaissant dans 24 et 25 stations constituent le groupe d'oiseaux ubiquistes qui ont réussi à coloniser tous les deux types de milieux de substitution. On retrouve ici des oiseaux très éclectiques comme Bulbucus ibis (Bui), Milvus migrans (Mim), Centropus senegalensis (Ces), Pycnonotus barbatus (Pyb), Tudoides plebejus (Tul), Corvinella corvina (Cor), Tchagra senegala (Tcs), Ploceus nigerrimus (Pli), Ploceus cucullatus et Turdus pelios (Tup) d'une part, et d'autre part des planctophages aériens présents au-dessus de tous les habitats en quête perpétuelle d'insectes: Hirundo semirufa (Hie), Hirundo senegalensis (Hin). Ces espèces peuvent rejoindre le groupe 6 des espèces ubiquistes.

En somme, on retrouve à travers le plan euclidien et la classification hiérarchique, une distribution des oiseaux identique à celle des stations. On remarque aussi que les communautés d'oiseaux de jachères sont numériquement plus importantes que celles des plantations.

\section{DISCUSSION}

Comparaison de la composition de l'avifaune inventoriée aux listes d'oiseaux d'autres auteurs dans l'aire d'étude

Il n'existe pas auparavant de liste spécifique aux milieux de substitution dans notre aire d'étude. Néanmoins, certains auteurs ont effectué des recensements d'oiseaux terrestres dans l'aire d'étude. Cela a servi de base de comparaison pour nos recensements.

Ainsi, toutes les 26 espèces typiques de jachère mentionnées déjà par Brunel (1958) ont été retrouvées. La buse à queue rousse d'Afrique (Buteo auguralis), le Grand indicateur (Indicator indicator) ont été signalés par Anciaux (1996) comme espèces de brousse (jachère) dans la région d'étude, mais n'ont été pas contactées. Ces deux espèces sont des oiseaux de savanes et sont citées par Green \& Sayer (1979) et Claffey (1995) plus au Nord du Bénin. Elles descendent certainement sporadiquement au Sud comme migrants africains ou erratiques.

La plupart des passereaux recensés dans cette étude avaient déjà été référencés par Anciaux (1996, 2000, 2002 a et b), Coubeou (1995), Waltert (1998), Akker (2003), mais la plupart d'entre eux sont rattachés aux milieux connexes des îlots de forêt du sud du Bénin. On peut alors postuler que ces passereaux recensés dans cette étude sont très indicatrices de la mutation des milieux forestiers. Ce sont donc des espèces qui ont réussi à s'accommoder des milieux de substitution après la déforestation récente qu'a connu le sud du Bénin. Leur suivi à long terme serait indispensable comme espèces indicatrices de l'altération des écosystèmes terrestres au sud du Bénin, surtout dans le contexte actuel des changements climatiques.

\section{Structuration des espèces et groupes écologiques}

Les structurations des espèces en groupes écologiques obtenues dans cette étude sont similaires à celles de Gottschalk et al., (2007), dans les savanes herbeuses de Serengeti. De telles structurations sont aussi obtenues par Kati \& Serkercioglu (2006) lors de l'étude sur la diversité, la structure écologique et la conservation des communautés d'oiseaux des paysages de la réserve de Diata en Grèce.

Lougbégnon et al., (2009) ont aussi débouché sur de telles associations 
écologiques des oiseaux dans leur travail sur les communautés d'oiseaux locales dans les mosaïques de végétation du Sud du Bénin. Ce qui prouve que l'arrangement écologique des oiseaux obtenu n'est pas une originalité des oiseaux des milieux de substitution étudiés. Cela met clairement en exergue le rôle bioindicateur des oiseaux.

La démarcation des plantations de Cocos nucifera des autres milieux tient sa justification $\mathrm{du}$ fait que ces plantations constituent des biotopes particuliers aujourd'hui dans cette partie du Bénin, parce que très proches de la côte et de la mangrove. Elles sont contiguës à des jachères anciennes, d'où le mélange d'avifaune de ses trois types de milieux (écotones). L'importance de la conservation d'un tel écosystème est primordiale dans notre aire d'étude pour espérer la sauvegarde de la biodiversité des oiseaux.

L'importance numérique du groupe d'avifaune des milieux herbacés et buissonnants tient $\mathrm{du}$ fait que dans l'aire d'étude, les jachères occupent les $75 \%$ des superficies dévouées aux domaines de substitution. De plus, elles peuvent offrir certaines conditions favorables, par exemple : les facilités de nidification à certains oiseaux ; d'autres oiseaux à tendance buissonnante trouveront suffisamment de structures végétatives à différents stades d'évolution pour continuer à nicher comme dans leur habitat naturel forestier. Ces jachères mettent également à la disposition des oiseaux une quantité appréciable de nourritures qui attirent beaucoup d'oiseaux omnivores, ce que les plantations monospécifiques n'arrivent pas à faire. De plus, étant donné que ces jachères forment souvent un continuum avec les champs, les oiseaux granivores et insectivores trouvent des grains (maïs, riz, etc.). En somme, ces jachères offrent à certains oiseaux des micro-habitats beaucoup plus favorables que les plantations.

Enfin, les espèces d'eau et les migrateurs paléarctiques ne sont pas vraiment inféodés à ces habitats car la présence des premiers n'est liée qu'à l'existence de l'eau dans ces milieux et les seconds n'utilisent ces habitats que pour une période de l'année.

\section{Conclusion}

Le travail a conclu à l'action déterminante de la végétation et de sa structure sur la diversité et la composition aviennes des jachères et plantations du Sud du Bénin. Les résultats de la distribution des oiseaux montrent l'existence de 6 groupes écologiques d'avifaunes. La couverture des différentes structures végétales de ces milieux a été déterminante pour la variation de la composition des stations investiguées. On déduit, l'existence de communauté d'oiseaux des plantations de Cocos nucifera, de Tectona grandis, de jachères arbustives et de jachères herbacée et buissonnante mais aussi, de communautés aviennes nettement ubiquistes pourvue qu'il existe de végétation. Les communautés d'oiseaux des plantations d'Elaeis guinnensis ne sont pas aussi bien individualisées comme celles des autres plantations. Elles ont tendance à s'assimiler aux jachères arbustives. Ces jachères détiennent une grande part de la communauté avienne de ces deux milieux.

Pour mieux conserver cette diversité de groupes d'oiseaux, le maintien des jachères sous plantations mixtes ou d'anciennes jachères est très souhaité car ces types d'habitats offriraient plus des conditions favorables à bon nombre d'espèces d'oiseaux de premier stades de colonisation et à certaines espèces forestières. Il serait souhaitable que l'on encourage de plus en plus les jachères anciennes dans le processus de maintien des milieux naturels pour la biodiversité dans cette partie du Bénin. Cette étude a montré que l'élément de base sur lequel il faut se fonder pour conserver l'avifaune est la variabilité de la structure végétative.

\section{REFERENCES BIBLIOGRAPHIQUES}

Akker M, van Den. 2003. Birds of Niaouli forest, southern Benin. Bulletin of the African Bird Club, 10(1): 1-17. 
Akoegninou A. 2004. Recherches botaniques et écologiques sur les forêts actuelles du Bénin. Thèse De Doctorat d'Etat en Botanique. UFR Biosciences Côte d'Ivoire. P. 326.

Anciaux MR. 1996. Aperçu de l'avifaune dans différents milieux de l'intérieur des terres du Sud-Bénin. Plateau d'Allada et sud de la dépression de la Lama. Cahiers Éthologie, 16: 79-98.

Anciaux MR. 2000. Approche de la phénologie de la migration des migrateurs intra-africains de l'intérieur des terres du sud-Bénin. Plateau d'Allada et sud de la dépression de la Lama. 1. Les nonCoraciiformes et les non-Passeriformes. Alauda, 68: 311-320.

Anciaux MR. 2002a. Approche de la phénologie de la migration des migrateurs intra-africains de l'intérieur des terres du sud-Bénin. Plateau d'Allada et sud de la dépression de la Lama. 2a. Les Coraciiformes et les Passeriformes. Alauda, 70: 203-211.

Anciaux MR. 2002b. Approche de la phénologie de la migration des migrateurs intra-africains de l'intérieur des terres du sud-Bénin. Plateau d'Allada et sud de la dépression de la Lama. 2b Discussion générale. Alauda, 70: 413-419.

Arbonier M. 2002. Arbres, Arbustes et Lianes des Zones Sèches d'Afrique de l'Ouest

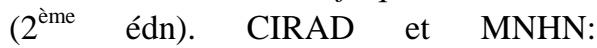
Montpellier.

Berlioz J. 1956. Étude d'une collection d'oiseaux du Dahomey. Bull. Mus. Natl. Hist. Nat., 23: 261-264.

Bersier LF, Meyer DR. 1995. Relationships between bird assemblages, vegetation structure, and floristic composition of mosaic patches in riparian forests. Rev. Ecol., 50: 15-33.

Bibby C, Burgess N, Hill D. 1992. Bird Census Techniques. B.T.O \& R.S.P.B. Academic press: London.

Blondel J. 1975. L'analyse des peuplements d'oiseaux, éléments d'un diagnostic écologique I. la méthode des échantillonnages fréquentiels progressifs
(E.F.P). Rev. Ecol, la Terre et la Vie, 29: 533-589.

Blondel J, Ferry C, Frochot B. 1970. La méthode des indices ponctuels d'abondance (I.P.A) ou des relevés d'avifaune par "stations d'écoute". Alauda, 38: 55-71.

Borrow N, Demey R. 2001. Birds of Western Africa ( $1^{\text {st }}$ edn). Christopher Helm: London.

Brunel J. 1958. Observations sur les oiseaux du bas-Dahomey. Oiseau et R.F.O., 28: 1-38.

Chappuis C. 2000. African Bird Sounds. Soc. Ornithol. : France, Paris, 15CD.

Chessel D, Debouzie D. 1983. Analyse des Correspondances et écologie : causes et conséquences du succès. Actes XII ${ }^{\mathrm{eme}}$ colloque "méthodes mathématiques appliquées en géographie". Besançon. 117-130 p.

Cordonnier, P. 1976. Etude du cycle annuel des avifaunes par la méthode des «points d'écoutes ». Alauda, 44(2) : 169-180.

Coubeou P. 1995. Diversité faunistique dans les différents biotopes de la forêt classée de la Lama. Thèse d'ingénieur agronome. FSA/UNB, Bénin. p 96.

Gottschalk ThK, Ekschmitt K, Bairlein F. 2007. Relationships between vegetation and bird community composition in grasslands of the Serengeti. Afr. J. Ecol., 45(4): 557-56.

Kati VI, Sekercioglu CH. 2006. Diversity, ecology structure and conservation of the landbird community of Dadia reserve, Greece. Diversity and Distributions, 12: 620-629.

Green AA, Sayer JA. 1979. The birds of Pendjari and Arli National Parks (Benin and Upper Volta). Malimbus, 1: 14-28.

Claffey PM. 1995. The birds of Bétérou area, Borgou Province, Republic of Bénin. Malimbus, 17: 63-84.

Lougbégnon OT. 2002. Le rôle de l'habitat sur la diversité de la faune avienne dans la zone subéquatoriale du Sud-Bénin. Mémoire de DEA en Gestion de 
l'Environnement. FLASH UAC Bénin. p 105.

Lougbégnon OT, Codjia JTC, Libois MR. (2009). Les communautés d'oiseaux locales dans les mosaïques de végétation du Sud-Bénin. Annales des Sciences Agronomiques du Bénin, 12(1): 67-84.

Martin J-L, Thibault J-Cl. 1983. Les oiseaux de la réserve naturelle de Scandola (Corse): inventaire et structure des peuplements. Bull. Ecol., 14(4): 279-296.

Prodon R. 1988. Dynamique des systèmes avifaune-végétation après déprise rurale et incendies dans les Pyrénées méditerranéennes siliceuses. Thèse de doctorat en sciences naturelles Université. Paris VI, p 333.

Prodon R, Lebreton JD. 1994. Analyses multivariées des relations espèces-milieu structure et interprètation écologique. Vie et Milieu, 44(11): 69-91.
Skowno AL, Bond WJ. 2003. Bird community composition in an actively managed savanna reserve, importance of vegetation structure and vegetation composition. Biodiversity and Conservation, 12: 2279-2294.

Sokpon N. 1995. Recherches écologiques sur la forêt dense semi-décidues de Pobè au Sud-est du Bénin: groupements végétaux, structure, régénération naturelle et chute de litière. Thèse de doctorat. Université Libre de Bruxelles. p.350.

Spitz F. 1982. Conversion des résultats d'échantillonnages ponctuels simple d'oiseaux en densités de population. Oiseaux Rev. Fr. Ornithol., 52: 1-14.

Walthert MH. 1998. Evaluation écologique intégrée de la forêt naturelle de la Lama en République du Bénin. Rapport sur l'inventaire des oiseaux. ONAB/KFW/GTZ. Cotonou, Bénin. p 36. 